\title{
Evidencias de neotectónica en el sector interno de la Faja Plegada y Corrida de Malargüe, valle del río Grande, Mendoza, Argentina
}

\author{
Neotectonic evidence in the inner section of the Malargüe Fold-and-Thrust Belt, Grande river valley, \\ Mendoza, Argentina
}

Diego A. Winocur ${ }^{1, *}$, Anahí Benítez ${ }^{1}$, Iván Barbero²

\begin{abstract}
Universidad de Buenos Aires, Facultad de Ciencias Exactas y Naturales, Departamento de Ciencias Geológicas. IDEAN (Instituto de Estudios Andinos UBA-CONICET). Intendente Güiraldes 2160, Ciudad Universitaria,1428, Ciudad Autónoma de Buenos Aires, Argentina

${ }^{2}$ Universidad de Buenos Aires, Facultad de Ciencias Exactas y Naturales, Departamento de Ciencias Geológicas. Intendente Güiraldes 2160. Ciudad Universitaria, 1428, Ciudad Autónoma de Buenos Aires, Argentina.
\end{abstract}

* Autor para correspondencia: (D. A. Winocur) winocur@gl.fcen.uba.ar

\section{Cómo citar este artículo:}

Winocur, D.A., Benítez, A., Barbero, I., 2021, Evidencias de neotectónica en el sector interno de la Faja Plegada y Corrida de Malargüe, valle del río Grande, Mendoza, Argentina: Boletín de la Sociedad Geológica Mexicana, 73 (2), A201220. http://dx.doi.org/10.18268/ BSGM2021v73n2a201220

Manuscrito recibido: 25 de Agosto de 2020 Manuscrito corregido: 30 de Octubre de 2020 Manuscrito aceptado: 8 de Noviembre de 2020

La revisión por pares es responsabilidad de la Universidad Nacional Autónoma de México.

\section{RESUMEN}

En este trabajo se exponen rasgos del paisaje que permiten inferir actividad neotectónica en el sector interno cordillerano (35으의 $\left.-70^{\circ} 15^{\prime} \mathrm{O}\right)$. A partir del trabajo de campo, se reconocieron depósitos lacustres caracterizados por arenas, limos y arcillas laminados, que registran sedimentación en una paleolaguna desarrollada durante un estadio del Último Máximo Glacial. La edad de los depósitos es interpretada a partir de su disposición en relación con una morena frontal atribuida a la última glaciación. A partir de la realización de un perfil sedimentario en los sedimentos lacustres se describen e interpretan estructuras asociadas a deformación en sedimentos blandos junto con fallas de origen tectónico, una de las cuáles, denominada falla El Seguro, presenta rechazo de $4 \mathrm{~m}$ medidos a lo largo del plano de falla y movimiento inverso. Los atributos morfológicos permiten interpretar que la deformación reconocida fue disparada por eventos sísmicos, proponiendo que la actividad asociada a dicha falla puede ser una posible fuente. La frecuencia y distribución de depósitos de remoción en masa mayores a $0.1 \mathrm{~km}^{2}$ y su ubicación respecto de las estructuras principales conocidas, y estructuras con actividad sísmica reciente y actual, permiten identificar una acumulación de deslizamientos en torno a éstas. Así, si bien han estado favorecidos por la litología, inclinación, pendientes y relajamiento pos-glacial del valle analizado, su disposición en el paisaje sugiere que la actividad sísmica precipitó el desarrollo de los movimientos en masa de mayor volumen. Finalmente, se propone que la actividad tectónica ha tenido lugar durante el Pleistoceno-Holoceno inclusive, con sismos de magnitud $\mathrm{Ms}_{\mathrm{s}} \geq 6$, de manera que la falla El Seguro podría ser una falla activa en la actualidad.

Palabras clave: licuefacción, sismos, neotectónica, megadeslizamientos, último máximo glacial, Malargüe.

\section{ABSTRACT}

This work exposes some features of the landscape that enable us to determine neotectonic activity in the internal sector of the cordillera

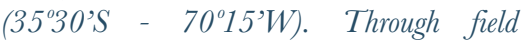
observations, there were recognized lacustrine deposits characterized by laminated sands, silts, and clays, which records the sedimentation in a paleolake during a stage of the Last Glacial Maximum. The age of the deposits is inferred from their position in relation to a frontal moraine attributed to the last glaciation. Based on a sedimentary profile in the lacustrine deposits, there are described soft-sediment deformation structures and tectonic faults, one of them named El Seguro fault, presents 4 $m$ of displacement measured along the fault plane, and an inverse movement. Morphological features indicate a seismic trigger, proposing the activity of the fault as a possible source. The frequency and distribution of $>0.1 \mathrm{~km}^{2}$ mass movement deposits and their location close to the active structures allow us to identify an accumulation of landslides around them. Although many factors have favored these movements as lithology, steep slopes and pos-glacial relief, their position in the landscape suggest that seismic activity accelerated the development of large mass movements. Finally, it is proposed that tectonic activity has taken place during the Pleistocene-Holocene inclusive, with earthquakes of magnitude $M s \geq 6$, so that $E l$ Seguro fault could be an active fault at present.

Keywords: liquefaction, earthquakes, neotectonics, megalandslides, last glacial maximum, Malargüe. 


\section{Introducción}

El levantamiento de la Cordillera de los Andes resulta de la subducción en el margen occidental sudamericano. La región de estudio se ubica en el segmento sur de los Andes Centrales (Gansser, 1973; Ramos, 1999), a los 35³0'S y 70¹5'O, al sur del segmento de subducción horizontal pampeano, bajo una subducción normal (Figura 1). El valle del río Grande se ubica en el sector interno de la faja plegada y corrida de Malargüe, cuya estructuración se desarrolló a partir del Cretácico Superior (Mescua et al., 2013; Tapia et al., 2015), y es considerada una región estructuralmente fósil en la actualidad (Kozlowski, 1993; Giambiagi et al., 2009, Folguera et al., 2015; entre otros). Durante el Pleistoceno, lenguas glaciarias cubrieron el valle principal y los tributarios, generando la extensa artesa por donde escurre el río Grande. Los avances glaciarios están representados por morenas agrupadas en el Drift Seguro, de edad estimada en 130,000 a 170,000 años, y en el Drift Valle Hermoso, correspondiente a la Última Glaciación (Espizua, 1998; Espizua et al., 2002). El paisaje se encuentra caracterizado además, por la presencia de depósitos correspondientes a deslizamientos y avalanchas de rocas de gran volumen, algunos de los cuales fueron identificados por Espizua y Bengochea (2002), quienes evaluaron el potencial riesgo geológico asociado. Según Antinao y Gosse, (2009) los deslizamientos mayores a $0.1 \mathrm{~km}^{2}$ pueden ser interpretados como disparados por eventos sísmicos a profundidades $\leq 20 \mathrm{~km}$ sobre la base de estudios realizados en la Cordillera Principal chilena. A su vez, según Keefer (1984), se requieren sismos de magnitudes $\geq 5$ para la ocurrencia de flujos de tierra, mientras que para la generación de avalanchas de rocas son necesarios sismos de magnitud Ms=6.5 (Rodríguez et al., 1999).

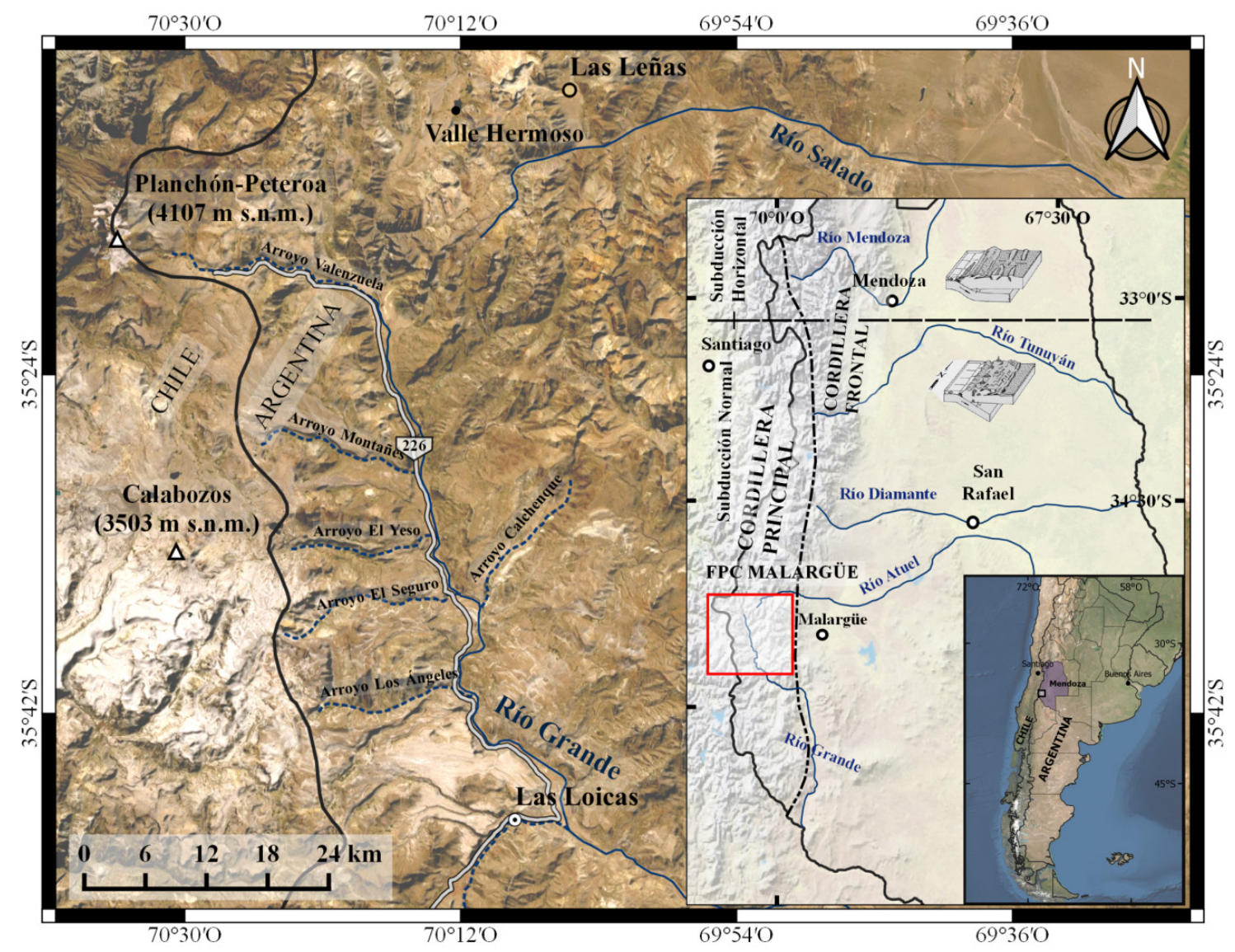

Figura 1 Ubicación de la zona de estudio. 
Trabajos recientes han reportado evidencias de actividad sísmica (Spagnotto et al., 2015; Villegas et al., 2016) y neotectónica en el sector chileno a los $35^{\circ} \mathrm{S}$ (Tapia et al., 2015; Giambiagi et al., 2019). Sin embargo, las deformaciones neotectónicas en sector argentino están circunscriptas a las zonas extracordilleranas y en el frente orogénico de deformación (Mescua et al., 2019; Colavitto et al., 2019).

En el presente trabajo se analizan las características y los procesos actuantes en el modelado del paisaje en el sector interno de la faja plegada y corrida de Malargüe, con el objetivo de identificar actividad tectónica reciente vinculada a fallamiento cuaternario, deformación de depósitos de origen glacilacustre y ocurrencia de megadeslizamientos en el sector septentrional del valle del río Grande y su implicancia en el análisis del riesgo sísmico en la región.

\section{Marco geológico}

El presente estudio se enfoca en el valle del río Grande, entre los $35^{\circ} 15^{\prime}$ y $35^{\circ} 45^{\prime} \mathrm{S}$ y los $70^{\circ} 15^{\prime} \mathrm{O}$, ubicado en el sector occidental de la provincia de Mendoza (Figura 1). Esta región forma parte de la Cordillera Principal (Yrigoyen, 1979) y se caracteriza por un basamento Permo-Triásico compuesto de volcanitas intermedias a ácidas pertenecientes al Grupo Choiyoi, que es cubierto por sedimentitas depositadas entre el Triásico Tardío al Paleógeno en una cuenca de retroarco denominada cuenca Neuquina (Legarreta y Uliana, 1999). El relleno de la cuenca se dio en tres estadios tectónicos: (i) una etapa de rift, durante el Triásico Tardío, representada por rocas volcánicas, volcaniclásticas y sedimentarias intercaladas del ciclo Precuyano, (ii) una etapa de subsidencia termal desde el Jurásico al Cretácico Temprano, en la que se depositaron sedimentitas marinas y continentales correspondientes a los Grupos Cuyo, Lotena, Mendoza y Rayoso durante varios eventos transgresivos $y$ regresivos, y (iii) el desarrollo de una cuenca de antepaís a partir del Cretácico, caracterizado por sedimentitas continentales y marinas de los Grupos Neuquén y Malargüe (Figura 2) (Giambiagi et al., 2008; Mescua et al., 2013).

Durante el Neógeno se produjo la principal fase de deformación compresiva en la zona, desarrollándose la faja plegada y corrida de Malargüe. El sector interno se caracteriza por un estilo estructural que combina fallas de basamento, algunas invertidas formando grandes anticlinales, con estructuras que resuelven acortamiento en la cubierta sedimentaria (Kozlowski et al., 1993; Giambiagi et al., 2005). Esta fase compresiva fue acompañada de magmatismo de arco, evidenciado en la zona por las volcanitas y cuerpos hipabisales de la Formación Huincán (Sruoga et al., 2020).

A partir del Plioceno, Ramos y Kay (2006), Folguera et al. (2008) y Folguera et al. (2009) han propuesto el desarrollo de fallas normales y volcanismo asociado en el sector interno de la faja plegada y corrida Malargüe, producto de un colapso orogénico durante el Plio-Cuaternario. Sin embargo, en el sistema de fallas El Fierro, valles del río Teno y Tinguiririca, a los $35^{\circ} \mathrm{S}$ en la vertiente chilena, los mecanismos focales de sismos recientes indican una cinemática transcurrente a transpresiva (Tapia et al., 2015; Giambiagi et al., 2019). En el frente orogénico se han registrado evidencias de reactivación de fallas y fallas cuaternarias con cinemática compresiva a transpresiva (Silvestro et al., 2005; Giambiagi et al., 2019; Mescua et al., 2019).

\section{Metodología}

Se realizó un relevamiento de campo que constó en la identificación, caracterización, interpretación y cartografiado de las principales geoformas y depósitos cuaternarios. El estudio de los depósitos de origen lacustre se llevó a cabo mediante el levantamiento de un perfil sedimentario a partir del cual se identificaron y describieron las litofacies y facies, y la distribución de los bancos con deformación a lo largo y ancho de toda la columna aflorante. 


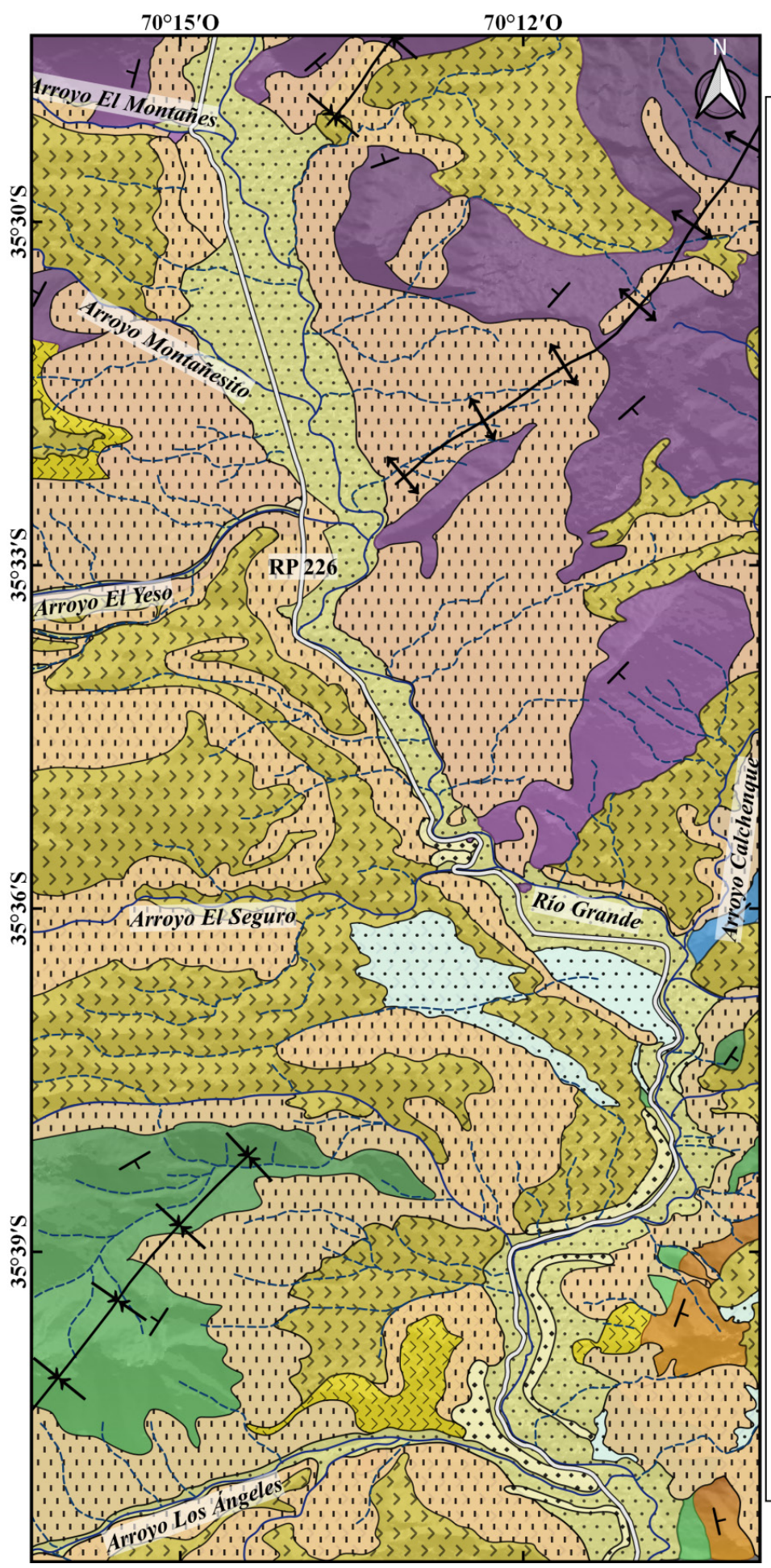

\section{Referencias}

Infraestructura

$\Longleftarrow$ Ruta Provincial No226

Cursos de agua

------ efimero

- permanente

Estructuras

$\underset{\downarrow}{\uparrow}$ Pliegue anticlinal

Columna estratigráfica

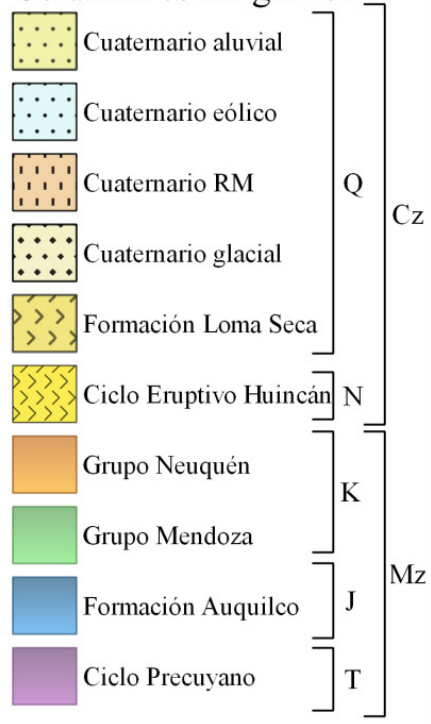

Escala

$1: 50.000$

$\begin{array}{llll}0 & 1 & 2 & 3 \mathrm{~km}\end{array}$

Figura 2 Mapa geológico del área de estudio. 
La descripción geomorfológica se ha enfocado en los depósitos morénicos y de remoción en masa circundantes a los depósitos lacustres. Los primeros condicionaron el desarrollo y evolución del depósito lacustre y el valle del río Grande durante el Pleistoceno-Holoceno, de manera que se realizó además un relevamiento con VANT (Mavic Pro) en los alrededores a la morena El Seguro para delimitar la extensión de los depósitos de interés. El análisis de los movimientos en masa se orientó al tipo de movimiento, área involucrada y distribución areal, ya que los que poseen área mayor a $0.1 \mathrm{~km}^{2}$ se vincularían a la ocurrencia de sismicidad como disparador (Antinao y Gosse, 2009). Para la identificación de sismos históricos, su ubicación, magnitud y profundidad se recurrió a la base de datos EHB (http://www.isc.ac.uk/ehbbulletin/search/ catalogue/) y de la Sociedad Geológica de los Estados Unidos (USGS) (https://earthquake. usgs.gov/earthquakes/search/).

Se complementó la interpretación geomorfológica mediante la utilización de imágenes satelitales Landsat-7, Landsat-8, Aster, y las disponibles en el software Google Earth y ARCGISEARTH. La información fue volcada en una base GIS, mediante la utilización del software libre Quantum GIS versión 3.2.3 (https://www. qgis.org) en la cual se han elaborado el mapa geológico y el mapa geomorfológico.

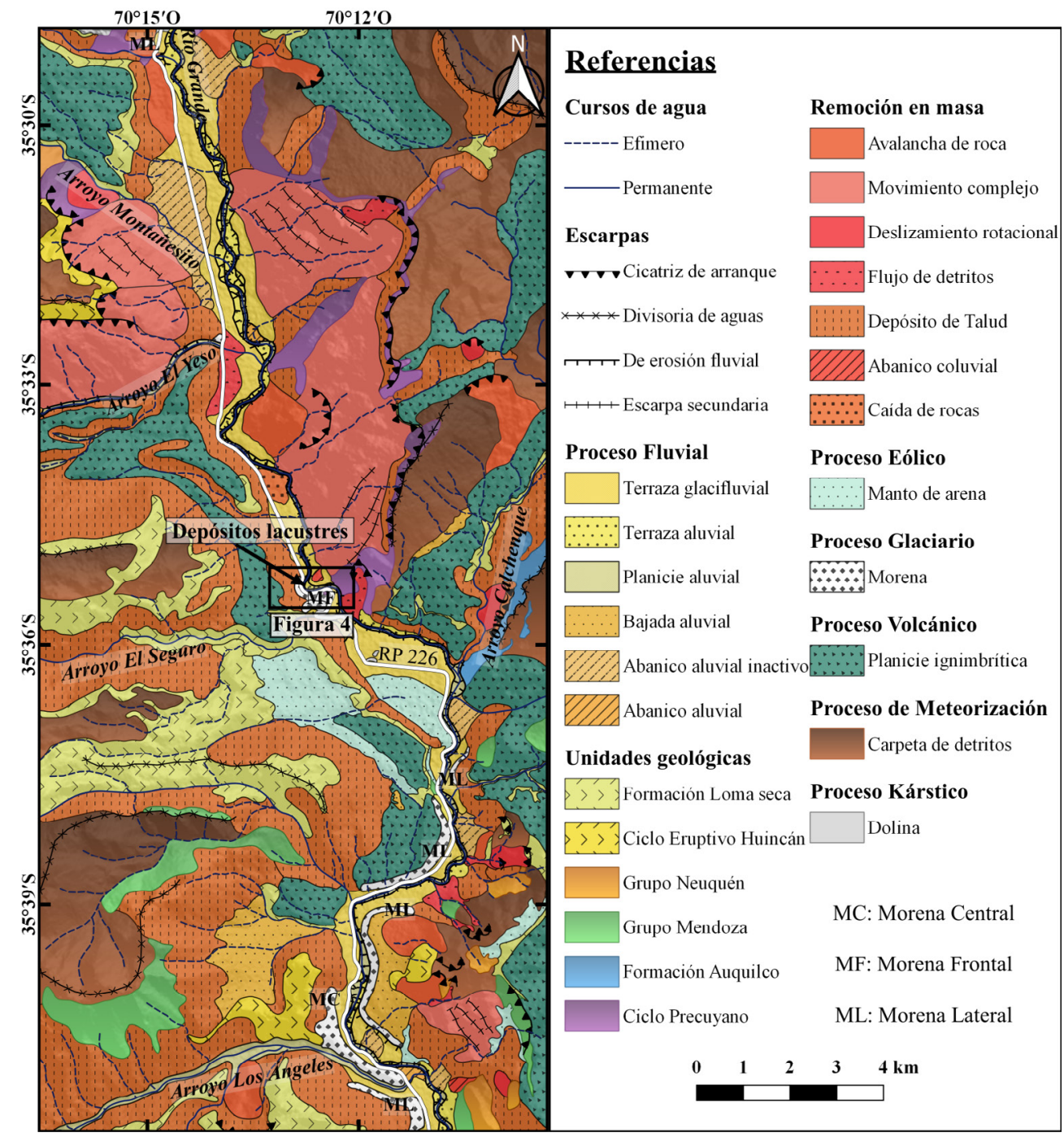

Figura 3 Mapa geomorfológico del área de estudio. Se indican los depósitos morénicos y la ubicación del depósito lacustre. 


\section{Resultados}

\subsection{ANÁLISIS DEL DEPÓSITO GLAGIARIO EL SEGURO}

El paisaje del sector central del valle del río Grande ha sido modelado principalmente por procesos glaciarios, de remoción en masa, fluviales y volcánicos (Figura 3). El relieve es montañoso, con alturas desde 1,700 m s.n.m. en el piso del valle hasta los 3,000 m s.n.m. en la cima de los cerros, y está caracterizado por pendientes mayores a $30^{\circ}$ hacia los extremos norte y sur, mientras que en el sector central presentan menor inclinación siendo las más conspicuas entre $5^{\circ}$ y $15^{\circ}$. La cuenca está conformada por un sistema colector principal de régimen permanente denominado río Grande, que es alimentado por varios arroyos tributarios y numerosos cursos efímeros, que le otorgan a la red de drenaje un diseño dendrítico.

Los depósitos morénicos preservados en cercanías al arroyo El Seguro quedan comprendidos dentro del Drift Seguro (Espizua, 1998). Al norte de la desembocadura del arroyo El Seguro, sobre el valle del río Grande, se identificó un depósito de till, cuya disposición curva, semicircular y perpendicular a la traza del río Grande permite inferir que se trata de una morena frontal (Figura 4 a y 4b). El depósito se encuentra parcialmente preservado en la margen occidental del valle, mientras que el sector noreste ha sido erosionado y ocupado por una terraza glacifluvial y dos terrazas fluviales (Figura 4b). El depósito posee continuidad lateral de manera paralela al río Grande con una orientación del flujo de la lengua glaciaria de aproximadamente $345^{\circ}$ y se apoya por sobre las rocas del ciclo Precuyano (Figura 5a y 5b), que presentan estrías, muescas y acanaladuras, evidenciando el efecto erosivo del glaciar. La morena tiene una altura de $60 \mathrm{~m}$ sobre la planicie aluvial actual, una extensión lateral de 700 m, y continúa como morena lateral hacia el norte. Se conforma de guijas a bloques con tamaños entre $4 \mathrm{~cm}$ a $2 \mathrm{~m}$

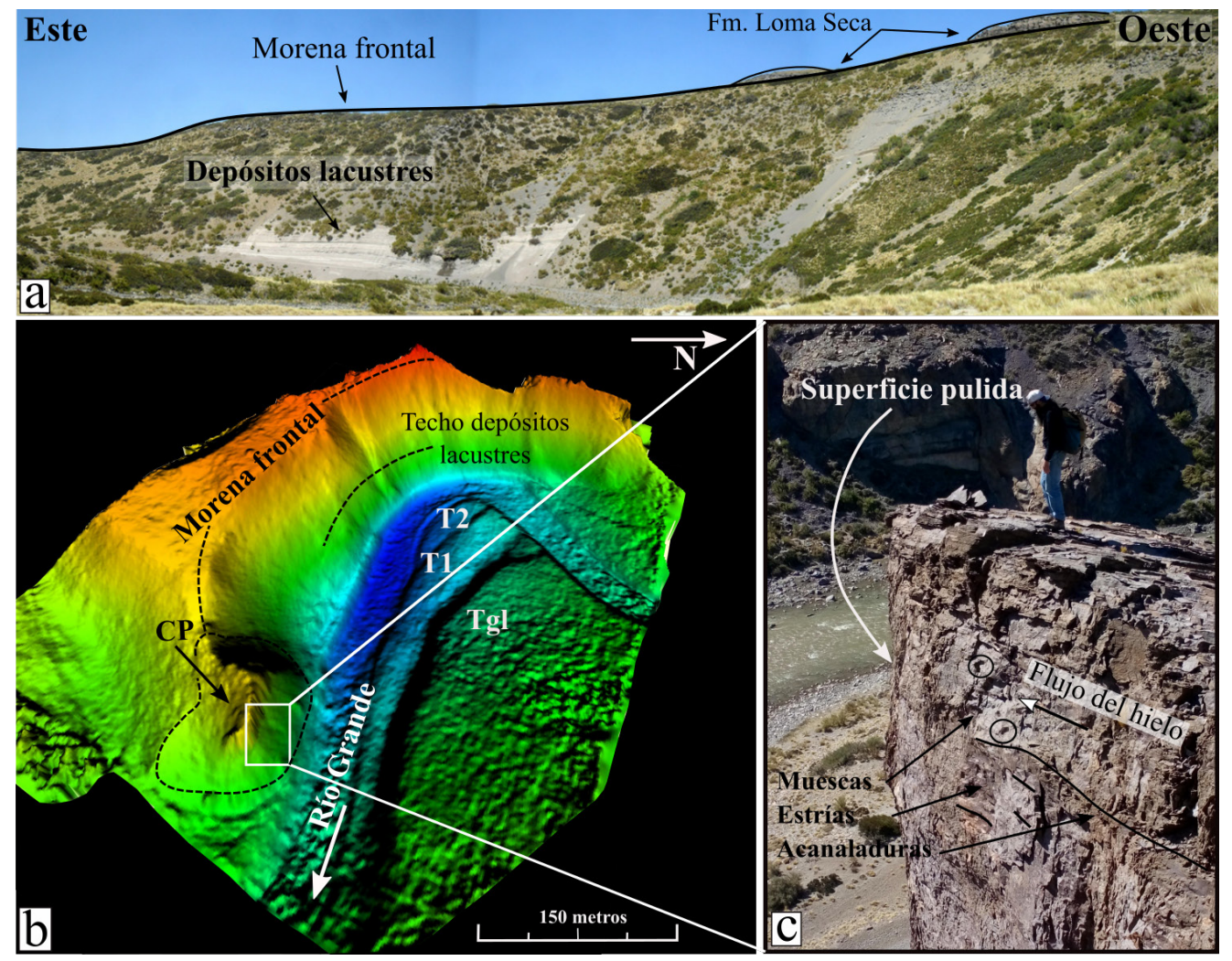

Figura 4 a. Fotografía de la morena frontal El Seguro y el depósito lacustre adosado. b. DEM obtenido a partir del VANT. Se indican los niveles de terrazas fluviales (T1 y T2) y glacifluviales (Tgl), la ubicación del depósito lacustre y las evidencias de erosión glaciaria en el ciclo Precuyano (CP). c. Microformas de erosión glaciaria. 
de diámetro, subredondeados, dispuestos en forma caótica en una matriz areno-limosa. Los clastos se componen principalmente de rocas volcánicas de la Formación Loma Seca y del ciclo Precuyano (Figura 5b y Figura 5c), y presentan abundantes estrías y facetamientos. Ubicado aguas arriba de la morena frontal, se encuentra adosado en contacto lateral un depósito lacustre de $33 \mathrm{~m}$ de espesor, medido mediante el DEM generado con el VANT (Figura 4b).

\subsection{ANÁLISIS DE DEPÓSITOS LAGUSTRES}

\subsubsection{DESCRIPCIÓN DE FACIES}

Los depósitos lacustres preservados en el sector interno de la morena frontal El Seguro tienen una potencia máxima aflorante de $33 \mathrm{~m}$. Consisten de limos y arcillas con intercalaciones de arenas finas a medias y ocasionales bancos de pocos centímetros de espesor de arenas gruesas y arenas guijarrosas. Para su caracterización, se levantó un perfil en la sección basal del depósito, debido a que en ese tramo se concentran las evidencias de deformación pos-depositacional (Figura 6).

De acuerdo al análisis sedimentario se definieron cinco facies: (A) Arenas, limos y arcillas laminados con deformación pos-depositacional, (B) Arenas y limos laminados, (C) Limos y arcillas interlaminados, (D) Limos y arcillas interlaminados con abundante deformación convoluta, y (E) Arenas con laminación ondulítica y horizontal en superficie inclinada. Sus características se muestran en la Tabla 1.

Facies A: Arenas, limosy arcillas laminados con deformación pos-depositacional: Esta facies se caracteriza por la alta deformación de los depósitos originales, que destruyen parcial o totalmente la estructura depositacional original, en tramos de varios metros de espesor (Figura 7a). Se trata de bancos laminados de limos y arcillas
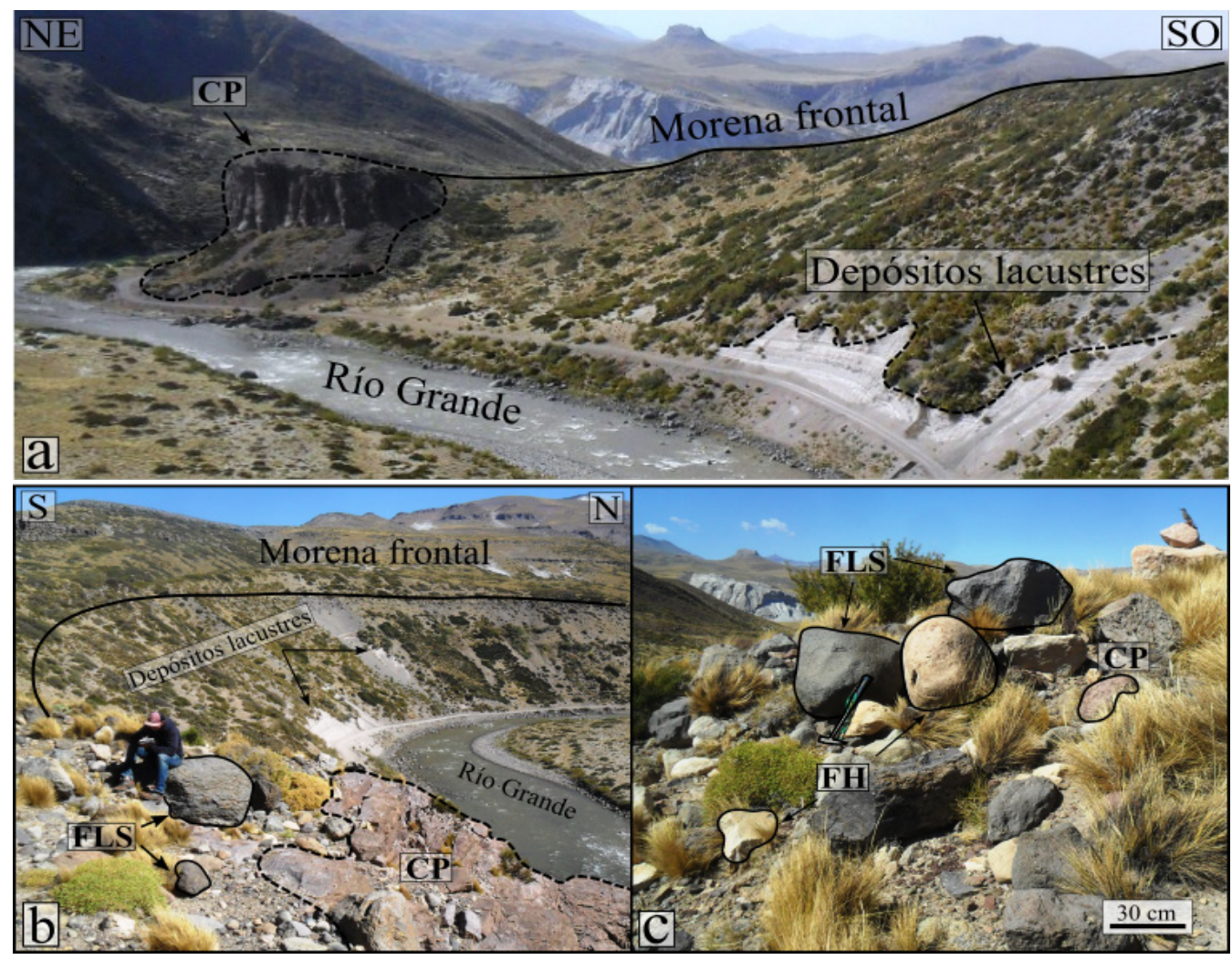

Figura 5 a. Vista al sur donde se observa la morena frontal El Seguro y su contacto con las rocas del ciclo Precuyano y los depósitos lacustres. b. Depósito morénico apoyado sobre rocas del ciclo Precuyano. c. Detalle de los componentes de la morena El Seguro. Los bloques pertenecen a la Formación Loma Seca (FLS), al ciclo Precuyano (CPC) y a la Formación Huincán (FH). 
Tabla 1. Facies definidas para la sección basal del depósito lacustre.

\begin{tabular}{|l|c|c|c|c|c|c|c|c|c|c|}
\hline & Total & \multicolumn{3}{|c|}{ Por quiebres de pendiente } & \multicolumn{5}{|c|}{ Por desplazamiento vertical } \\
\hline & - & $\mathrm{n}=1$ & $\mathrm{n}=2$ & $\mathrm{n}=3$ & $\mathrm{n}=4$ & $\mathrm{~h}<2.5$ & $2.5<\mathrm{h}<5$ & $5<\mathrm{h}<7$ & $7<\mathrm{h}<9$ & $10<\mathrm{h}<13$ \\
\hline Media & 4.6 & 3.2 & 5.7 & 7 & $10.16^{*}$ & 1.3 & 3.7 & 5.9 & 8.2 & 11 \\
\hline$\pm \sigma$ & 3,1 & 2.7 & 2.9 & 1.4 & $0.012^{*}$ & 0.5 & 0.7 & 0.5 & 0.5 & 0.012 \\
\hline Moda & 3.6 & 2.2 & 3.6 & 5.8 & & 0.5 & 3.6 & 5.8 & 7.5 & 10.7 \\
\hline Mediana & 3.7 & 2.2 & 4.6 & 6.6 & $10.16^{*}$ & 1.3 & 3.7 & 5,8 & 8.3 & 10.9 \\
\hline Asimetría & 0.8 & 1.7 & 0.8 & 0.4 & & 0.2 & 0.09 & -0.06 & -0.14 & 0.4 \\
\hline Pruebas & & Por quiebres de pendiente & & Por desplazamiento vertical \\
\hline Media & - & 2.2 & 3.6 & 7 & 10.7 & 1.3 & 3.5 & 5.7 & 8.1 & 10.7 \\
\hline$\pm \sigma$ & - & 0.8 & 0.4 & 1.2 & 0.7 & 0.4 & 0.25 & 0.35 & 0.4 & 0.6 \\
\hline
\end{tabular}

que alternan esporádicamente con láminas de arenas finas a muy finas.

Sin embargo, en algunos sectores la deformación es total, lo que le otorga un aspecto masivo. Así, la pervasiva deformación de los sedimentos no permite registrar cambios granulométricos o en la disposición de las láminas de arena a lo largo de la columna. El contacto superior con la facies B es neto, aunque obliterado por el desarrollo de calcos de carga y flames. Las estructuras deformacionales consisten en pseudonódulos y ball and pillow de arenas de la facies $\mathrm{B}$, de tamaños variables entre 0.1 y 0.6 m de diámetro, y deformación convoluta de aspecto caótico, con zonas donde se reconocen pliegues redondeados, en punta o cuspidados (Figura 7a y 7e). En algunos sectores se identificaron pequeñas fallas inversas con rechazos centimétricos que cortan a los bancos ya deformados (Figura 7e).

Interpretación: El ambiente de depositación corresponde a un sector de muy baja energía, que permitió la decantación a partir de suspensión del material fino. Las láminas de limo grueso y los esporádicos bancos arenosos podrían indicar flujos densos subácueos en una zona relativamente profunda, por debajo de la acción del oleaje.

Facies B: Arenas y limos laminados: Consiste en láminas horizontales de gran continuidad lateral, formando bancos de hasta $0.6 \mathrm{~m}$ de espesor, dispuestas en alternancia. Se componen de arenas finas a medias masivas, y se presentan en láminas horizontales en la base, que gradan hacia limos y arcillas, en menor proporción (Figura 7b). Algunos bancos de arenas, con espesores que alcanzan los $5 \mathrm{~cm}$, presentan laminación ondulítica de oleaje. Ocasionalmente, intercalan en la base, bancos masivos de hasta $0.5 \mathrm{~cm}$ de espesor de arenas con sábulos y guijas, y escasa continuidad lateral.

Interpretación: La elevada proporción de arenas finas a medias, a veces con laminación ondulítica, indican depositación en aguas someras en condiciones de baja energía. Los bancos masivos de arenas gravillosas son interpretados como flujos gravitatorios asociados a la elevada pendiente de los depósitos morénicos circundantes. 


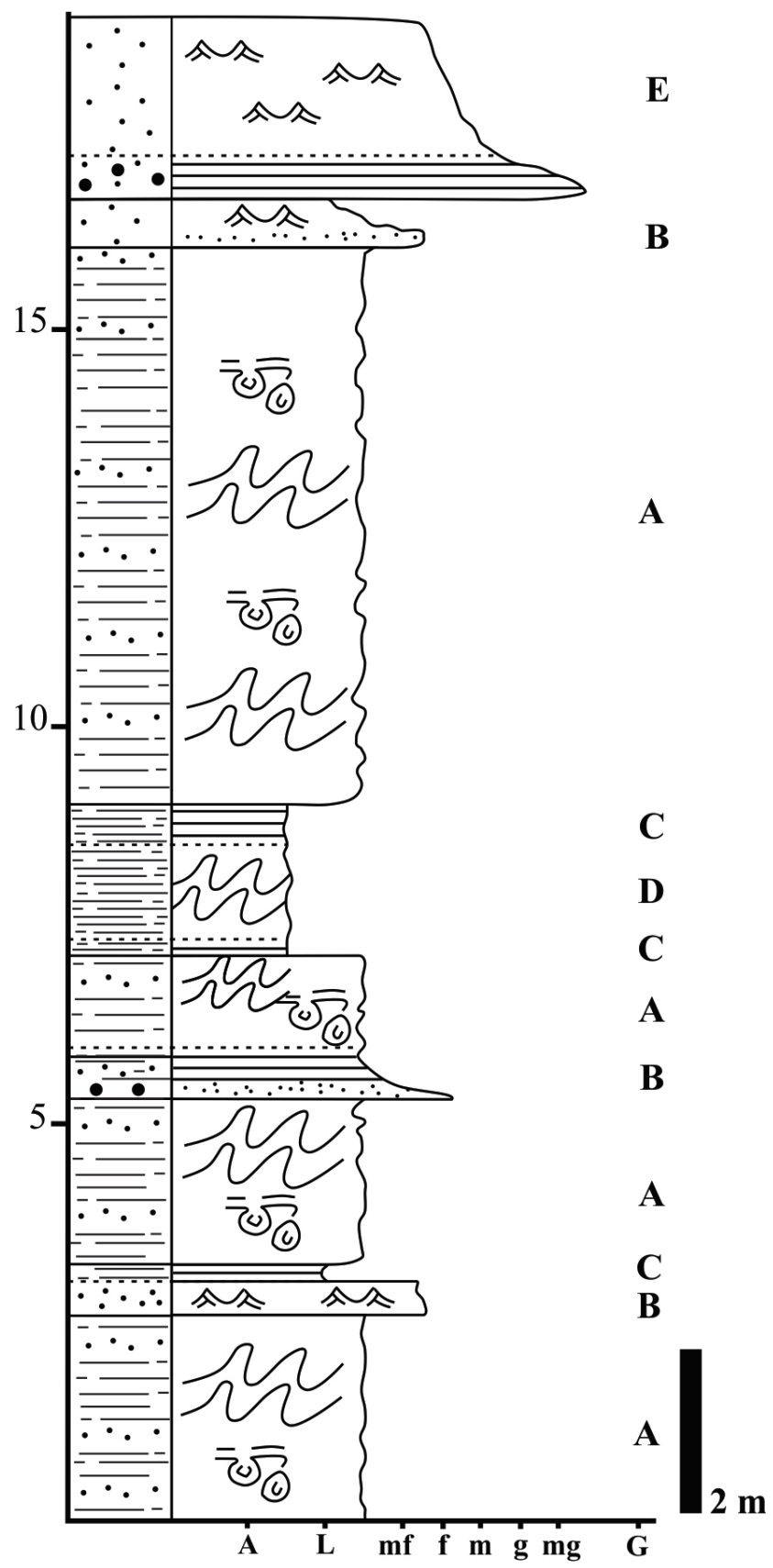

\begin{tabular}{l} 
Laminacion horizontal \\
\hline \hline Laminación convoluta \\
Ball and pillow, pseudonódulos \\
y/o calcos de carga
\end{tabular}

Figura 6 Perfil sedimentológico del depósito lacustre. Ver ubicación en la figura 3. 
La laminación de material muy fino implica condiciones de depositación por decantación y flujos subácueos de baja energía. El conjunto es interpretado como un ambiente relativamente somero, que ocasionalmente se encontraba sometido a la influencia de oleaje de buen tiempo.

Facies C: Limos y arcillas interlaminados: Se trata de bancos milimétricos muy continuos lateralmente de limos gruesos a finos y arcillas. Intercalan bancos de hasta $0.5 \mathrm{~cm}$ de espesor de arenas finas, masivas. El contacto es transicional con la facies A, mientras que con la facies $\mathrm{D}$, si bien es no erosivo, se encuentra afectado por la deformación de los bancos suprayacentes, generando un aspecto ondulado (Figura 7c).

Interpretación: La presencia exclusiva de limos y arcillas en alternancia, indican depositación en un ambiente muy tranquilo, por debajo de la acción del oleaje. Esta asociación de litofacies conforma ritmitas depositadas a mayor profundidad que las facies sucesivas, con ocasionales depósitos de flujos densos.

Facies D: Limos y arcillas interlaminados con abundante deformación convoluta: Esta facies se compone de limos y arcillas altamente deformadas y masivas. La estructura original corresponde, al menos parcialmente, a láminas de ambas granulometrías intercaladas. Sin embargo, no es posible identificar si esta laminación se mantiene en todo el espesor de los paquetes. En algunos sectores se distingue laminación convoluta que involucra además arenas finas laminadas, internamente masivas, en bancos de entre 0.1 y $0.3 \mathrm{~cm}$ de espesor. Se identifican algunos pseudonódulos y ball and pillow, de hasta $5 \mathrm{~cm}$ de diámetro, que internamente se componen de láminas horizontales deformadas compuestas de limos gruesos y arenas muy finas (Figura 7d).

Interpretación: Considerando la granulometría y estructuras originales preservadas, la depositación tuvo lugar en un ambiente subácueo de muy baja energía. El origen de las intercalaciones de arenas y paquetes arenosos laminados se interpretan como depósitos asociados a flujos subácueos o depositados bajo condiciones de baja energía con aporte de arena, en un sector a menor profundidad.
Facies E: Arenas con laminación ondulítica y horizontal en superficie inclinada: Esta facies sobreyace a la facies B en forma transicional. Se caracteriza por la presencia dominante de arenas finas a medias, en bancos tabulares y continuos, de entre 0.05 y $0.1 \mathrm{~m}$ de espesor. Hacia la base domina la laminación horizontal, intercalada con delgados bancos limosos, que gradan a laminación ondulítica de oleaje ascendente, y laminación horizontal o inclinada de bajo ángulo de arenas medias a gruesas, y ocasionalmente, sábulos y guijas con matriz arenosa formando bancos de hasta $3 \mathrm{~cm}$ de longitud. Hacia el tope de esta facies dominan los bancos de arenas finas a medias con laminación ondulítica de oleaje (Figura 7f).

Interpretación: La granulometría y estructuras reconocidas indican un aumento en la energía del medio respecto a la facies D. La conspicua presencia de laminación ondulítica de oleaje indica depositación de arenas en la faja costera por encima del nivel de olas de buen tiempo. La presencia de arenas medias a gruesas y gravas finas horizontales da cuenta de una costa con disponibilidad de material gravilloso proveniente del aporte fluvial y morénico circundante.

\subsubsection{CONSIDERACIONES PALEOAMBIENTALES}

La secuencia de depósitos descritos anteriormente se encuentra en contacto lateral con el depósito morénico generado previamente y yace sobre depósitos glacifluviales. Domina la sedimentación clástica de limos y limos arcillosos laminados dispuestos en ritmitas, evidenciando un ambiente de depositación subácuea de muy baja energía. La alternancia de finas capas de arenas y arenas limosas masivas, sugieren la recurrencia de flujos de fondo subácueos, mientras que los depósitos masivos de acotada extensión lateral de arenas con sábulos y gravilla evidencian la esporádica ocurrencia de flujos gravitatorios, producto de las elevadas pendientes y disponibilidad de detrito suelto proveniente de la morena y de las laderas circundantes. La sección basal de la sucesión representa un sector de relativa profundidad pero con periodos de exposición al retrabajo por acción 


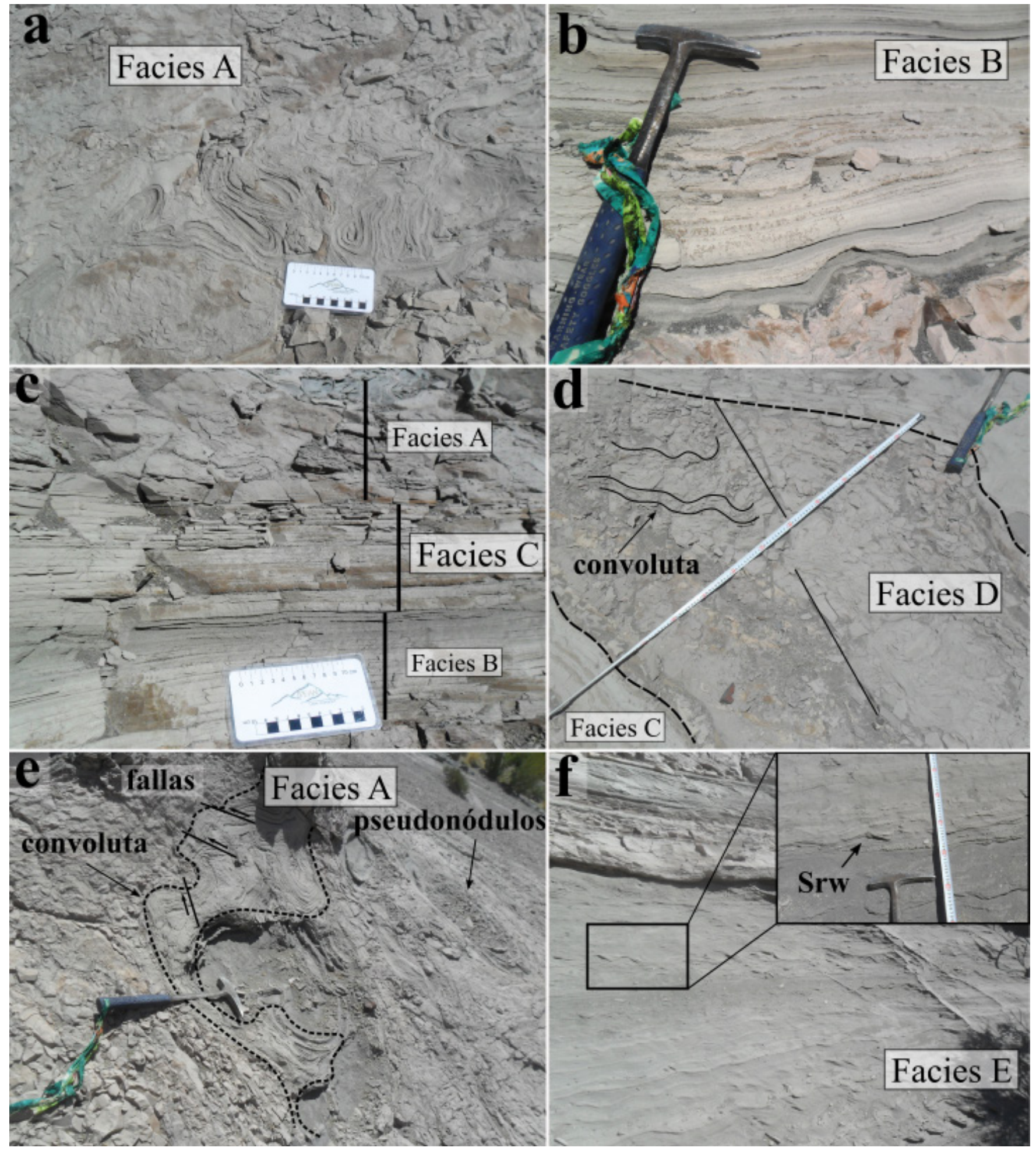

Figura 7 Detalles de bancos del depósito lacustre. a. Deformación convoluta en la facies A. b. Laminación horizontal de limos y arenas de la facies B. c. Detalle de estructuras depositacionales de las facies A, B y C. d. Limos y arcillas masivos producto de deformación posdepositacional, en la facies D. e. Bancos muy deformados de la facies A. Se indican pseudonódulos arenosos, estratificación convoluta y fallas inversas con doble vergencia f. Estructuras depositacionales en las arenas de la facies E, compuesta por areniscas con laminación ondulítica de oleaje (Srw). Hacia la base hay laminación horizontal e inclinada de bajo ángulo. 
del oleaje. La sección intermedia, conformada por las facies $\mathrm{C}$ y $\mathrm{D}$, representa una etapa de mayor profundización del sistema lacustre, mientras que hacia la sección superior queda registrada una progresiva somerización. Esto evidencia oscilaciones en el nivel de profundidad del paleosistema lacustre. Además, su desarrollo se vio propiciado por el depósito morénico, que actuó como barrera natural generando un endicamiento, al tiempo que la lengua glaciaria se encontraba en retroceso aguas arriba, y alejada de la laguna. Esto se evidencia en que a lo largo del afloramiento lacustre y en el perfil levantado, no se han observado rasgos indicativos de contacto directo con el hielo glacial.

\subsubsection{CONSIDERACIONES DE LA DEFORMACIÓN}

La deformación de la sucesión sedimentaria analizada puede dividirse en dos grupos: (i) estructuras deformacionales en sedimentos saturados en agua, y (ii) estructuras frágiles de escala de centímetros a metros. Las estructuras dentro del grupo (i) más frecuentes corresponden a deformación convoluta, calcos de carga, estructuras flames, pseudonódulos y ball and pillow. El origen de las estructuras deformacionales en sedimentos blandos frecuentemente se asocia al proceso de licuación, mediante el cual un aumento en la presión de los fluidos porales genera que los sedimentos saturados en agua pierdan momentáneamente su cohesión y se deformen (Maltman, 1994). La abundancia de pliegues volcados, cuspidados y las geometrías caóticas y discontinuas en bancos de las facies A y D sugieren escape de fluidos (Figura 8). Por otro lado, la depositación de capas de sedimentos con distinta permeabilidad implica diferentes tasas de disminución de la porosidad, por lo que capas de mayor densidad pueden yacer sobre capas de menor densidad, generando un gradiente inverso. Los calcos de carga y estructuras asociadas se producen en estos casos (Owen, 2003). La reducción de la presión efectiva puede darse por distintos motivos. Los más frecuentes, a la escala analizada en este trabajo, corresponden a sobrecarga dada por rápida sedimentación y/o el peso de glaciares, generación de gases a partir del decaimiento de materia orgánica, y el paso de ondas sísmicas (Collinson, 2005). Entre ellos, considerando el contexto evolutivo del paisaje, los mecanismos más viables corresponden a la rápida sedimentación y los eventos sísmicos. Sims (2013) realizó similares observaciones en sistemas lacustres con rasgos semejantes, atribuyendo un origen sísmico a las estructuras deformacionales.

La ocurrencia de deformación de sedimentos blandos no se ha desarrollado en todo el depósito, sino en capas discretas dentro de la sección inferior, donde se realizó el perfil. Esto implica que los procesos que dispararon la deformación se dieron de modo recurrente pero en un periodo acotado de tiempo. Así, las estructuras deformacionales
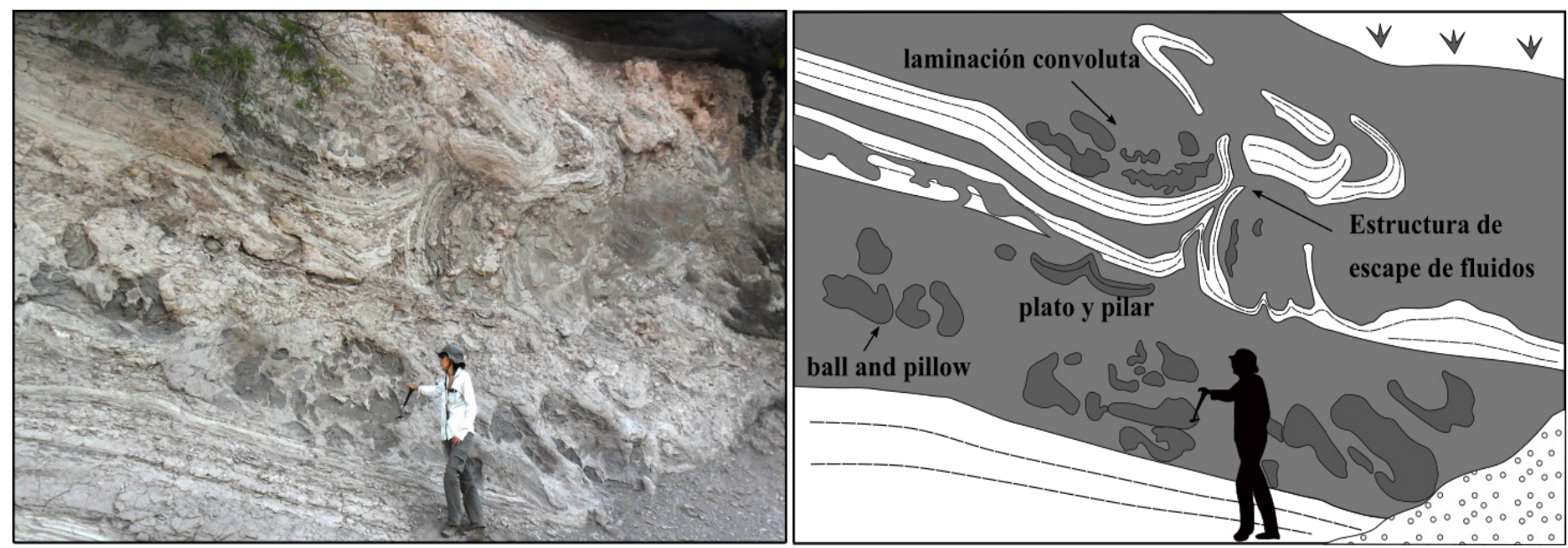

Figura 8 Fotografías de detalle de las estructuras deformacionales presentes en algunos bancos del depósito lacustre. 
resultantes pueden ser complejas, ya que si el proceso disparador ocurrió repetidamente a intervalos de tiempo cortos, los sedimentos ya disturbados pueden continuar deformándose (Van Loon et al., 2016).

Las estructuras de deformación frágil se encuentran representadas por una falla inversa de mayor jerarquía denominada falla El Seguro (Figura 9a), pequeñas fallas inversas (Figura 9c) y escasas fallas normales con rechazos aparentes de pocos centímetros (Figura 9b). La falla El Seguro posee un azimut aparente $220^{\circ} / 25^{\circ}$, coincidente con la orientación de las principales estructuras observadas en la región (Figura 2 y 11) y un rechazo aparente medido a lo largo del plano de falla de $4.10 \mathrm{~m}$ (Figura 9a). Si bien no se observan evidencias de la falla en el relieve, existe un cambio de pendiente sobre el depósito morénico en dirección de la falla (Figura 5). Sin embargo no hay evidencias claras de que se trate de una escarpa ya que se encuentra cubierta por depósitos de talud y vegetación. La base del depósito lacustre se encuentra afectada por la falla El Seguro, aunque la continuidad de su traza en profundidad queda cubierta por depósitos de talud producto de las altas pendientes y la removilización del material debido a la realización de la ruta provincial 226 (Figura 9a).

Las fallas de menor escala en general presentan movimiento inverso, con rechazos centimétricos (Figura 9b y 9c). Se desarrollan tanto en bancos deformados previamente como en bancos sin
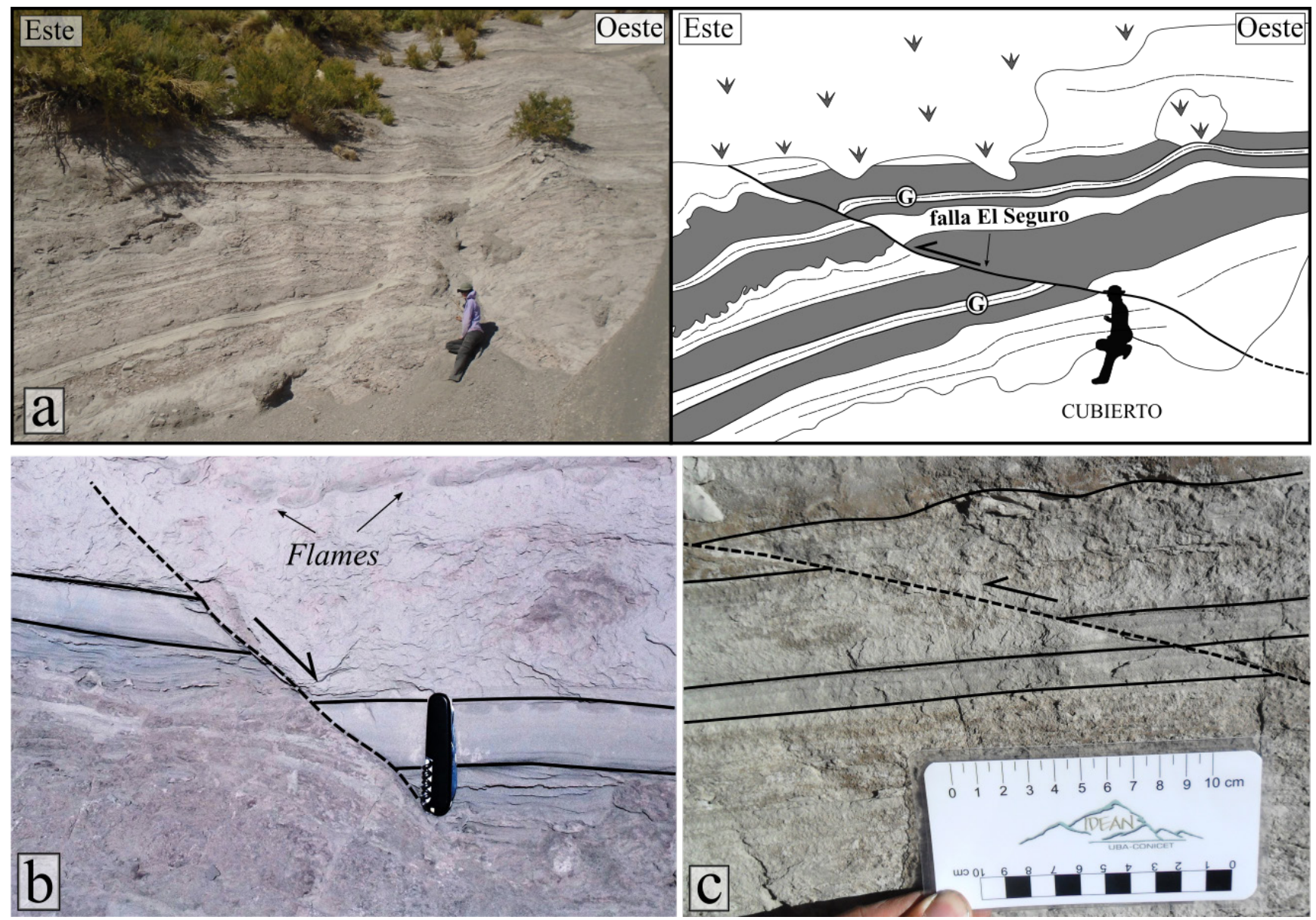

Figura 9 a. Fotografía de la falla El Seguro, afectando a los depósitos lacustres, y su interpretación. El rechazo de 4,1 m fue medido a lo largo del plano de falla tomando al banco guía "G". b. Fotografías de ejemplo de falla normal con rechazos centimétricos. c. Fotografías de ejemplo de falla inversa con rechazos centimétricos. 
deformar. Su distribución es heterogénea, aunque su frecuencia disminuye al aumentar la distancia a la falla El Seguro. Por lo tanto, se interpreta que estas estructuras secundarias están relacionadas a la actividad tectónica de la falla, ya que se observan a lo largo de todo el depósito y que su actividad es posterior a los eventos de licuación.

\subsection{DISTRIBUGIÓN DE DESLIZAMIENTOS}

En el valle del río Grande en su sector norte y central, son frecuentes los depósitos de remoción en masa, que según su morfología y sedimentología pueden clasificarse, en orden decreciente de abundancia como deslizamientos, flujos y movimientos complejos (Varnes, 1978; Hungr et al., 2014). Si bien la litología involucrada, su orientación estructural y pendientes, y la relajación pos-glaciaria de laderas son factores que influyen en su desarrollo, los disparadores pueden ser distintos. Los movimientos de poco volumen son más frecuentes y son generados en su mayoría por eventos climáticos, mientras que aquellos de mayor jerarquía se asocian a eventos sísmicos (Antinao y Gosse, 2009). De esta manera, fueron identificados 59 depósitos de remoción en masa con un área superior a 0.1 $\mathrm{km}^{2}$, que se distribuyen en zonas específicas. En estos sectores afloran las areniscas y volcanitas del ciclo Precuyano, afectadas por estructuras extensionales invertidas generando anticlinales y sinclinales que favorecen el desarrollo de deslizamientos planares (Figura 10).

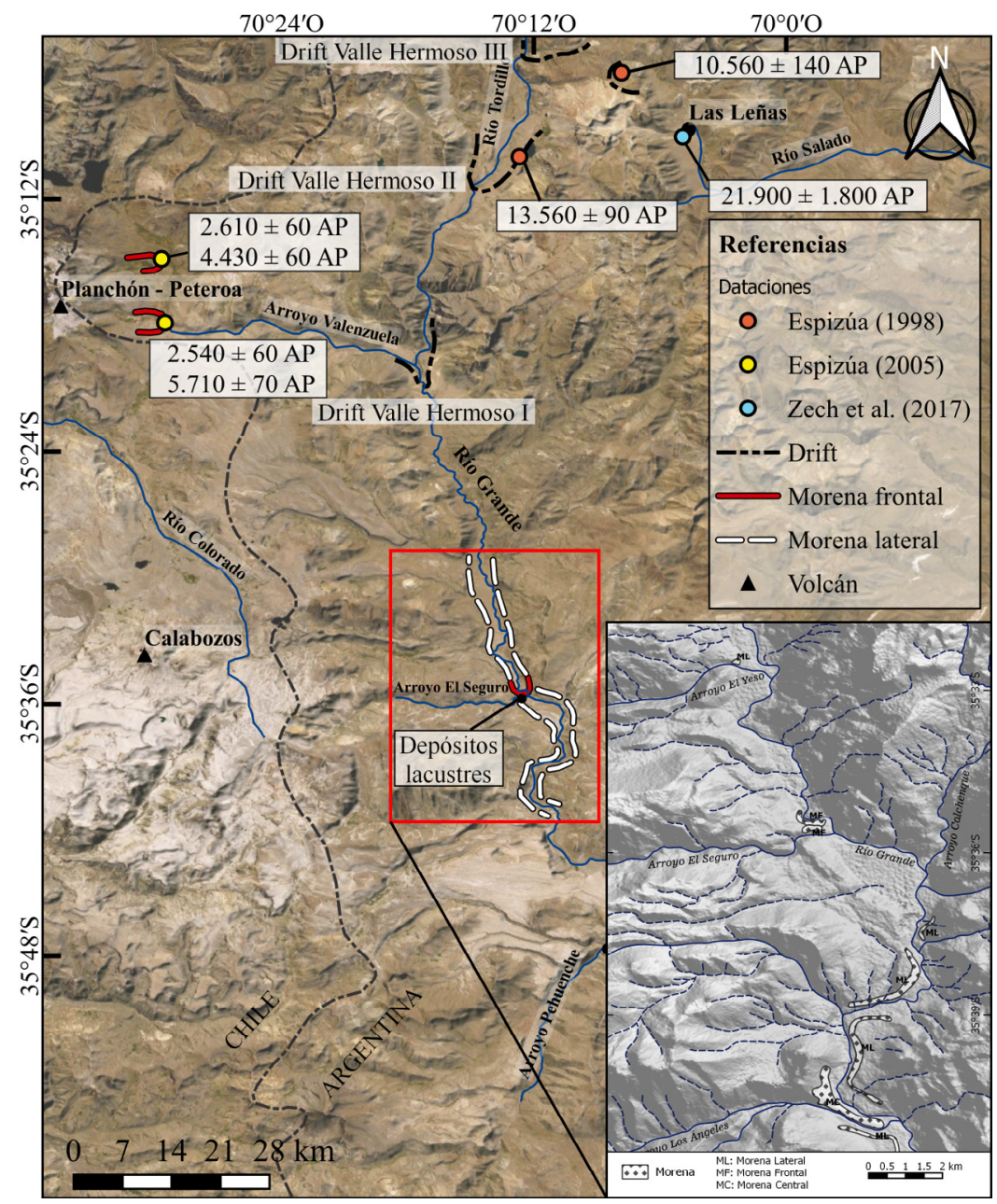

Figura 10 Distribución de depósitos de remoción en masa con área mayor a $0.1 \mathrm{~km}^{2}$. Se indican las estructuras principales involucradas y la ubicación de epicentros de sismos recientes, ocurridos a profundidades menores a $35 \mathrm{~km}$. 


\section{Discusión}

\subsection{ESTIMAGIÓN DE LA EDAD DE LA DEFORMAGIÓN GUATERNARIA}

En este sector de la Cordillera Principal mendocina, las evidencias de avances glaciarios cuaternarios se restringen a las cabeceras y sector central del valle del río Grande, al valle Hermoso, inmediatamente al norte de la zona de estudio, al valle del arroyo Las Leñas, hacia el noreste y a los valles Peñon y Azufre en inmediaciones del volcán Planchón-Peteroa (Figura 11). La evolución glaciaria y paraglacial del valle del río Grande, y las edades numéricas de los avances glaciarios en la región se limitan a la propuesta de Espizua, (1998) y Espizua et al. (2002). Considerando esto y las evidencias presentadas en este trabajo, se interpreta una posible edad de la deformación asociada a la historia geomorfológica y evolutiva de los depósitos glaciarios.

El Drift Seguro, que contiene a la morena frontal identificada en el presente trabajo, no posee dataciones numéricas y su edad fue asignada al Pre-Wisconsin (Espizua, 1998), con base en características del depósito y mediante la correlación de

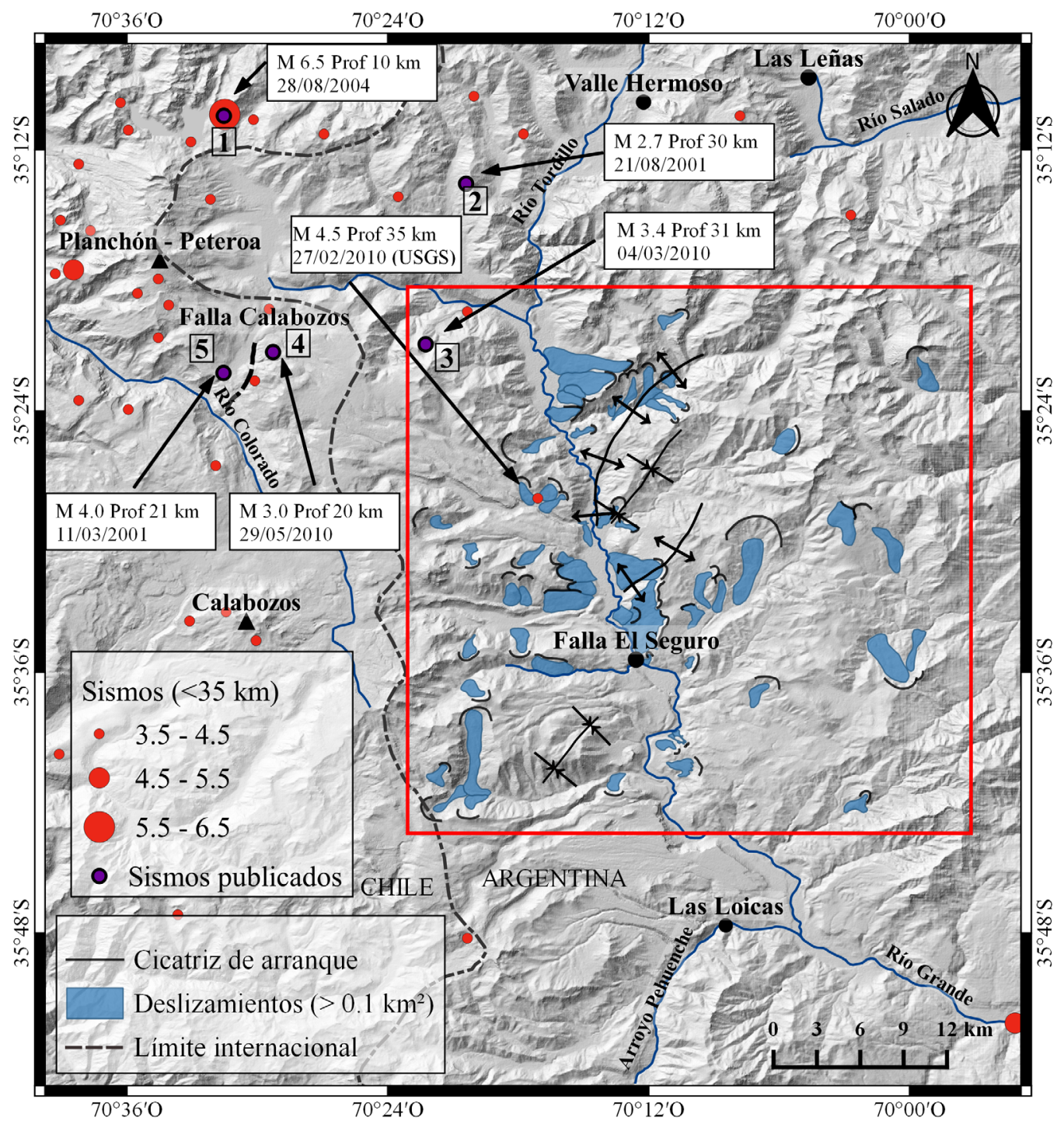

Figura 11 Ubicación y edades disponibles de los depósitos glaciarios asociados al Último Máximo Glacial en la Cordillera Principal mendocina, entre los $35^{\circ}$ y $36^{\circ} \mathrm{S}$. En el recuadro se indican los depósitos morénicos reconocidos en este trabajo. Todas las edades están referidas con anterioridad al presente (AP). 
dicho drift con el de Punta de Vacas en el valle del río Mendoza a los $32^{\circ} \mathrm{S}$. Este depósito presenta edades de trazas de fisión de 360,000 $\pm 36,000$ años (Espizua, 1993). Sin embargo, trabajos recientes desestiman la existencia del Drift Punta de Vacas a base de edades entre 25,000 y 23,000 años obtenidas mediante nucleidos cosmogénicos en depósitos glacifluviales ubicados a alturas entre 1,900 y 1,750 m s.n.m. (Fauqué et al., 2009, 2014). Depósitos asociados a la Última Glaciación han sido registrados en el valle de Las Leñas, al noreste del valle del río Grande (Figura 11), donde se preservan niveles morénicos con edades máximas de 21,900 años, obtenidas mediante isótopos cosmogénicos a alturas de 2,200 m s.n.m. (Zech et al., 2017). A su vez, en el valle Hermoso, el análisis de horizontes de turba dentro de las morenas de los drifts Valle Hermoso II y III, arrojó edades ${ }^{14} \mathrm{C}$ de $13,560 \pm 30$ A.P. y 10,560 \pm 140 A.P. presentándose a alturas de 2,170 y 2,500 m s.n.m. respectivamente (Espizua et al., 2002). Las morenas observadas en inmediaciones del volcán Planchón-Peteroa (Figura 11), arrojan edades entre $5,710 \pm 70$ y $2,540 \pm 60$ A.P. mediante el método ${ }^{14} \mathrm{C}$ en horizontes de turba ubicadas a 2,200 m s.n.m aproximadamente (Espizua 2005; Espizua y Pitte, 2009; Durán et al., 2016).

Debido a la ausencia de una edad numérica del Drift Seguro, se tomaron en cuenta las alturas de las morenas y sus posiciones relativas dentro del valle, las edades numéricas disponibles y las descripciones de campo realizadas para estimar su edad relativa. Sobre esta base, se observa que la morena frontal El Seguro se ubica a una altura de 1,750 m s.n.m., altitud cercana a la observada en el Drift Punta de Vacas datado en 25,000 y 23,000 A.P. (Fauqué et al., 2014). Por otro lado, las edades más cercanas ubicadas en las cabeceras de la cuenca del río Grande, corresponden al Drift Valle Hermoso III y II, cuyas cotas se encuentran a 2,170 y 2,500 m s.n.m y edades que oscilan entre $\sim 13,500$ y 10,500 A.P. Esto implica que el retroceso glaciario queda manifestado en la edad de los depósitos, los que son progresivamente más jóvenes hacia el norte (Figura 11). Las morenas analizadas en Las Leñas por Zech et al. (2017), pueden ser cronológicamente correlacionables con las de El Seguro aunque se encuentren a una cota superior. Esto puede ser explicado considerando las variaciones en las condiciones climáticas, la orientación y la extensión del valle del río Salado con respecto al valle del río Grande (Figura 10). Finalmente los datos cronológicos presentados por Espizua (2005), Espizua y Pitte, (2009) y Durán et al. (2016), en inmediaciones del volcán Planchón-Peteroa representan un avance glaciario durante el Holoceno a alturas de 2,200 m s.n.m., de manera que corresponde a un avance glaciario posterior y más reciente al analizado en el presente trabajo (Figura 10). De esta manera, se considera que el depósito morénico El Seguro corresponde a un retroceso glaciario perteneciente al Último Máximo Glacial (UMG).

La formación de la morena frontal El Seguro y las sedimentitas de origen lacustre que se disponen en contacto lateral con la misma, permiten inferir un período de estabilidad glacial durante el paulatino retroceso glaciario. A su vez, los depósitos lacustres no presentan evidencias de haber estado en contacto directo con el hielo, por lo que se interpreta que la lengua glaciaria se encontraba retirada hacia las cabeceras del valle. Esto implica que los depósitos lacustres se asocian a una laguna generada a partir del endicamiento del valle del río Grande generado por la morena frontal El Seguro, y alimentada por un curso fluvial surgido del agua de derretimiento de la lengua glacial situada aguas arriba. Se estima que la misma se ha desarrollado en un período comprendido entre los $\sim 20,000$ y los $\sim 13,590$ años, considerando las edades de los depósitos glaciarios en Valle Hermoso y Las Leñas. La deformación pos-depositacional en sedimentos blandos se acota a dicho periodo, durante el cual la ocurrencia de eventos sísmicos que pudieron haber disparado la deformación fueron recurrentes. Las evidencias de comportamiento frágil que presenta la falla El Seguro y su rechazo aparente, permiten inferir que su movimiento se dio a partir de más de un evento sísmico, ocurrido con posterioridad al retroceso glaciario, a la deformación por licuación 
Tabla 2. Datos de sismos recientes y cercanos a la zona de estudio.

\begin{tabular}{|l|c|c|c|c|c|c|c|}
\hline Eventos & & $\mathbf{1}$ & $\mathbf{2}$ & $\mathbf{3}$ & $\mathbf{4}$ & $\mathbf{5}$ & $\mathbf{6}$ \\
\hline Experimento 1 & & & & & & \\
\hline Agrupamiento por DV & DV & 1.3 & 3.5 & & 5.7 & 8.1 & 10.7 \\
\hline Experimento 2 & $\pm \sigma$ & 0.4 & 0.35 & & 0.35 & 0.4 & 0.6 \\
\hline Agrupamiento por DV & DV & 1.3 & 3.1 & 4.7 & & 8.1 & 11.3 \\
\hline Experimento 3 & $\pm \sigma$ & 0.4 & 0.3 & 0.4 & & 0.4 & 0.5 \\
\hline Agrupamiento por DV & DV & 1 & 3 & 4.5 & 6.6 & 8.3 & 10.7 \\
\hline
\end{tabular}

de los sedimentos y a la posterior colmatación de la laguna, tal que los mismos se hayan encontrado consolidados al momento de la deformación.

Por consiguiente, la falla El Seguro ha tenido actividad durante el Pleistoceno-Holoceno, y posiblemente durante los últimos 10,000 años, considerándose una falla activa según la clasificación propuesta por Costa et al. (2000).

\subsection{LOGALIZACIÓN DE LOS SISMOS DISPARADORES DE LA DEFORMAGIÓN}

La repetición de estructuras deformacionales de sedimentos blandos a lo largo del depósito lacustre dan idea de ciclicidad o recurrencia de eventos, los que podrían llegar a tener, en este contexto geomorfológico y geológico, dos tipos de fuentes. Un posible origen corresponde a sismos vinculados al rebote isostático producto del retiro del glaciar. Estudios recientes en el hemisferio Norte, relacionado al retiro glacial del campo de hielo escandinavo durante el UMG, sugieren que sismos de magnitudes entre 4.5 a 5 pueden generar licuación con una recurrencia de eventos sísmicos de entre 100 a 150 años, registrados a partir de niveles deformados en depósitos lacustres (Van Loon et al., 2016). Otros autores asignan un origen tectónico a las estructuras de licuación halladas en secuencias lacustres genéticamente vinculadas a glaciares del UMG, como las observadas en el norte de Alemania (Pisarska-Jamroży et al., 2018; Grube, 2019). En los Andes Centrales la relación existente entre el rebote isostático y la generación de sismos es poco clara y no ha sido estudiada. Las masas de hielo desarrolladas en el UMG en la zona de estudio corresponden a lenguas glaciarias de valle de acotada extensión areal, por lo que se estima que el rebote isostático no sería suficiente para explicar la generación de estructuras de deformación frágil, ni la licuación de los depósitos lacustres.

La deformación de los depósitos lacustres también podría vincularse al glaciotectonismo, sin embargo ésta se da principalmente en ambiente subglacial, en depósitos de till basal, o en ambiente proglacial, asociado a morenas frontales. Esta deformación solo puede ser posible debido a un avance del glaciar que supere la resistencia del material a la deformación (Aber et al., 1989; Bennett y Glasser, 2009). Sin embargo, la morena El Seguro es interpretada como una morena de retroceso que representa un único estadio de estabilización, sin evidencias de deformación en sus depósitos, ni de un avance glaciario. Esta 
interpretación es coincidente con Espizúa, (1998) y Espizua et al. (2002) quienes no han encontrado evidencias de reavances glaciarios, sino depósitos de drifts asociados a deglaciación.

Finalmente, las características del depósito lacustre evidencian que el mismo no ha tenido contacto alguno con el hielo glacial, por lo que no ha estado sujeto a esfuerzos compresivos que sean producto de un empuje glaciario. Por las razones mencionadas anteriormente se descarta un posible origen glaciotectónico de la deformación de los depósitos lacustres. Así, el desarrollo de estas estructuras con rechazos mayores a $4 \mathrm{~m}$, es vinculado a un origen tectónico resultante de un campo de esfuerzos compresivo, con el esfuerzo principal mínimo $\sigma 3$ vertical.

La distancia entre las estructuras deformacionales o con evidencias de licuación y el epicentro del terremoto han sido ampliamente discutidas a lo largo de todo el mundo. Se han sugerido que sismos de $M=6$ pueden generar licuación en un radio de $9 \mathrm{~km}$ desde el epicentro, mientras que aquellos con $\mathrm{M}=7$ pueden extender su radio a 70 $\mathrm{km}$ (Seed, 1969). Del mismo modo, se han indicado que sismos de magnitud entre 5 y 7 pueden generar licuación de sedimentos en un radio de $25 \mathrm{~km}$ del epicentro (Audemard y De Santis, 1991), en tanto que sismos de magnitudes entre 6 y 8 pueden gatillar este proceso en radios entre 40 y $100 \mathrm{~km}$, respectivamente (Obermeier, 1994; Moretti et al., 1995).

La evidencia más cercana de fallas activas descritas en la zona, corresponde a la falla Calabozos, ubicada $35 \mathrm{~km}$ al oeste, que posee evidencias de actividad pos-pleistocena y reciente (Farías et al., 2009; Tapia et al., 2015) (Figura 11). Santibáñez et al. (2019) han propuesto que las fallas ubicadas dentro del dominio del arco volcánico, poseen un potencial sísmico moderado, estimado en magnitudes de entre 6.2 a 6.7. Estas fallas, según estos autores, poseen tasas de movimiento altas, en torno a $7.0 \mathrm{~mm} / \mathrm{año}$, con una recurrencia comprendida entre cientos a miles de años. Por otra parte, el evento sísmico histórico más cercano a la región con evidencia de licuación es el terremoto de Villa
Atuel ocurrido en 1929 en San Rafael (Lünkenheimer, 1929). El epicentro de este terremoto de Ms=6.8 se ubica aproximadamente a $180 \mathrm{~km}$ al este del valle del río Grande (Cisneros y Bastías, 1993). Asociado a este evento, se han documentado numerosos cráteres y surgimiento de aguas calientes $\left(18^{\circ} \mathrm{C}\right)$.

Considerando la ocurrencia y registro de sismos, su magnitud, profundidad y ubicación, y los mecanismos focales, magnitudes y profundidades indicados por otros autores para la zona (Comte et al., 2008; Villegas et al., 2016; Spagnotto et al., 2015), se puede establecer una relación con las deformaciones aquí descritas (Tabla 2). Así, el sismo registrado en 2001 de $\mathrm{M}=4.0$ asociado a la falla Calabozos (Villegas et al., 2016), se encuentra dentro del radio de susceptibilidad a la ocurrencia de procesos de licuación en la zona. Por lo tanto, es una posible fuente de sismos disparadores de la deformación en los depósitos lacustres durante el Pleistoceno-Holoceno.

La actividad de la falla El Seguro también puede haber disparado deformación en los sedimentos blandos lacustres, cuando el tip de la ruptura se encontraba aún por debajo de los niveles deformados, al tiempo que los sedimentos permanecían saturados en agua. De esta manera, la licuación pudo ser gatillada por sismos de poca magnitud, recurrentes en el tiempo durante el Pleistoceno-Holoceno.

La relación entre movimientos de remoción en masa y la sismicidad también ha sido objeto de estudio. Los criterios de evaluación sugeridos se asocian a: (i) La existencia de sismicidad registrada en la zona; (ii) la magnitud mínima requerida para generar un deslizamiento; (iii) la distribución de deslizamientos en relación con una falla activa o una zona sísmicamente activa; (iv) el área afectada por los deslizamientos en relación con la magnitud del sismo; (v) la máxima distancia del deslizamiento generado respecto al epicentro, considerando su magnitud, y a la ruptura en superficie de la falla; y (vi) evidencias de licuación asociados con los deslizamientos (Keefer, 1984; Crozier, 1992; Rodriguez et al., 1999). 
La identificación y distribución de depósitos de remoción en masa en la zona permiten determinar que 59 deslizamientos presentan un área mayor a $0.1 \mathrm{~km}^{2}$, y se encuentran concentrados en dos sectores específicos. En el sector norte, las distancias a epicentros de sismos recientes con magnitudes superiores a 4.5 se encuentran en torno a la decena de kilómetros, y a su vez distan a $20 \mathrm{~km}$ de la falla Calabozos. Sumado a ello, las estructuras anticlinales y sinclinales presentes en este sector han ejercido un rol condicionante en el desarrollo de estos movimientos, ya que se disponen paralelamente al rumbo de los mismos (Figura 11). En el sector sur, la distancia de los deslizamientos con relación a la falla El Seguro es menor a $1 \mathrm{~km}$ (Figura 11). La presencia de depósitos con estructuras de licuación relacionadas con la falla El Seguro permite inferir que la actividad de dicha estructura pudo también disparar movimientos rápidos de remoción en masa. Así, el conjunto de características indicadas en torno a la distribución areal de los grandes deslizamientos ubicados en la región sur, pueden interpretarse como generados a partir de sismos vinculados a la falla El Seguro, mientras que los ubicados en el sector norte son vinculados a las mismas fuentes generadoras de sismos en la actualidad, tales como los sistemas de fallas El Fierro y Calabozos.

\subsection{CONSIDERACIONES SOBRE EL RIESGO SÍSMICO}

En este trabajo se exponen evidencias que permiten reconocer a la falla El Seguro e inferir que puede ser activa en la actualidad. Considerando el rechazo mayor a $4 \mathrm{~m}$ a lo largo del plano de falla, la deformación generada por procesos de licuación observada en los depósitos lacustres, y teniendo en cuenta registros sísmicos someros de magnitud Ms=6.5 cercanos a la zona de estudio (Comte et al., 2008), resulta importante el análisis detallado de la actividad de esta estructura para ser considerada en el análisis del riesgo símico de la provincia de Mendoza y del departamento de Malargüe en particular, ya que reviste un potencial riesgo sísmico para la región. El mismo se asocia a la ubicación de la falla respecto de la localidad de Las Loicas, la cual dista menos de $25 \mathrm{~km}$ y está habitada por 187 personas distribuidas en 55 hogares (Carrizo et al., 2018). Sin embargo, existen 33 puestos dispersos en la región, 2 de los cuales se ubican a menos de $2 \mathrm{~km}$ de la falla. Otro de los elementos en riesgo es la ruta provincial 226, ya que su traza atraviesa la falla y es la única vía de acceso al paso fronterizo argentino-chileno denominado Vergara y su puesto de gendarmería correspondiente. Es de destacar además, la inminente construcción del proyecto de aprovechamiento multipropósito Portezuelo del Viento (UNGuyo, 2017), cuya cota máxima estará en los 1,630 m s.n.m. y distará $12 \mathrm{~km}$. aguas abajo de la falla El Seguro. Esto resultará en la elevación del nivel freático regional, ocasionando el incremento de las presiones porales en los depósitos porosos, tales como tefras y cenizas, aumentando la posibilidad de que frente a un shock sísmico se desestabilicen las laderas adyacentes al proyecto propuesto como así también alterar el normal funcionamiento del mismo.

\section{Conclusiones}

En el presente trabajo se dan a conocer rasgos geomorfológicos y deformacionales en sedimentos cuaternarios, que evidencian actividad neotectónica en las estribaciones occidentales de la Faja Plegada y Corrida de Malargüe en la provincia de Mendoza. Se identificaron depósitos lacustres de una paleolaguna desarrollada durante el Último Máximo Glacial, endicado por una morena frontal, con niveles deformados por licuación disparada por eventos sísmicos. Estos depósitos, además, se encuentran afectados por una falla inversa denominada El Seguro con un rechazo aparente a lo largo de su plano de falla mayor a los $4 \mathrm{~m}$ que podría haber estado asociada a sismos de magnitudes $\mathrm{Ms} \geq 6$. Se interpreta además, que la fuente sismogénica responsable de la licuación en los depósitos de la paleolaguna se puede asociar a dicha falla 
cuando el tip de la ruptura se encontraba aún por debajo de los sedimentos deformados, aunque no se descarta que haya sido producto de otras fuentes sismogénicas cercanas. La actividad sísmica en la zona también pudo desencadenar el desarrollo de grandes movimientos de remoción en masa, favorecidos por la litología, inclinación y relajación de laderas pos-glaciarias. Así, se infiere que la falla El Seguro podría ser una falla activa desde el Pleistoceno-Holoceno a la actualidad. Esta identificación es de relevancia respecto del conocimiento del riesgo sísmico en la región del valle del río Grande, ya que además de ubicarse la localidad de Las Loicas, está propuesta la construcción del proyecto multipropósito Portezuelo del Viento. Por lo tanto, se recomienda la realización de un estudio detallado e integral del potencial peligro sísmico de la región.

\section{Agradecimientos}

Los autores desean expresar su agradecimiento a la Dra. Laura Perucca por la invitación a participar del presente volumen especial. También a la Universidad de Buenos Aires por haber otorgado el subsidio UBACYT 2018-20020170200399BA y al Dr. Victor Durán director del proyecto PICTO-UNCUYO 2016. Finalmente hacemos extensivo el agradecimiento a los árbitros, el Dr. Felipe Tapia y un árbitro anónimo, por sus valiosas recomendaciones que ayudaron a mejorar el manuscrito. Esta contribución lleva el número R-394 del IDEAN.

\section{Referencias}

Aber, J.S., Croot, D.G., Fenton, M.M., 1989, Glaciotectonic landforms and structures. Kluwer Academic Publishers, Dordrecht. $200 \mathrm{p}$.

Antinao, J. L., Gosse, J., 2009, Large rockslides in the Southern Central Andes of Chile (32$\left.34.5^{\circ} \mathrm{S}\right)$ : Tectonic control and significance for Quaternary landscape evolution:
Geomorphology, 104, 117-133. https://doi. org/10.1016/j.geomorph.2008.08.008

Audemard, A., De Santis, F., 1991, Survey of liquefaction structures induced by recent moderate earthquakes: Bulletin, International Association Engineering Geologists, 44, 4-16. https://doi. org/10.1016/0148-9062(92)92820-3

Bennett, M.R., Glasser, N.F., 2009, Glacial geology: ice sheets and landforms. John Wiley \& Sons. London, 377 p.

Carrizo H. G., Godoy, N., Moiso Fogal, G., 2018, Informe Censo Socio-Económico: población afectada por cota de embalse y poligonal de afectación. Proyecto "Aprovechamiento Multipropósito Portezuelo del Viento". Provincia de Mendoza, $32 \mathrm{p}$.

Cisneros, H., Bastías, H., 1993, Neotectónica del Borde oriental del Bloque de San Rafael (resumen), en $12^{\circ}$ Congreso Geológico Argentino y $2^{\circ}$ Congreso de Exploración de Hidrocarburos, Mendoza, Argentina, Actas, 3, 270-276.

Colavitto, B., Sagripanti, L., Fennell, L., Folguera, A., Costa, C., 2019, Evidence of Quaternary tectonics along Río Grande valley, southern Malargüe fold and thrust belt, Mendoza, Argentina: Geomorphology, 346, 1-59. https://doi.org/10.1016/j. geomorph.2019.06.025

Collinson, J., 2005, Sedimentary processes. Post-Depositional Sedimentary Structures, en Selley, R., Cocks, L.,Plimer, I. (eds), Encyclopedia of Geology. Elsevier Academic, 602-611. https://doi:10.1016/ b0-12-369396-9/00467-6

Comte, D., Farías, M., Charrier, R., González, A., 2008, Active Tectonics in the Central Andes: 3D tomography based on the aftershock sequence of the 28 August 2004 shallow crustal earthquake (resumen), en $7^{\circ}$ International Symposium on Andean Geodynamics, Niza, Francia, ISAG, 160-163.

Costa, C., Machette, M., Dart, R., Bastías, H., Paredes, J., Perucca, L., Tello, G., Haller, K., 2000, Map and Database of Quaternary 
Faults and Folds in Argentina, Denver: U.S.A., U.S. Geological Survey, Report 00-0108, 75 p. https://doi.org/10.3133/ofr00108

Crozier, M.J., 1992, Determination of paleoseismicity from landslides, en Landslides: Proceedings of the Sixth International Symposium, Christchurch, New Zealand. 1173-1180.

Durán, V., Winocur, D., Stern, G., Garvey, R., Barberena, R., Peña Monné. J, y Benitez, A., 2016, Impacto del volcanismo y glaciarismo holocénicos sobre el proceso de poblamiento humano de la cordillera sur de la provincia de Mendoza (Argentina): una perspectiva geoarqueológica: Revista Intersecciones en Antropología, 4, 33-46.

Espizua, L.E., 1993, Quaternary glaciations in the Río Mendoza valley, Argentine Andes: Quaternary Research, 40, 150-162. https:// doi.org/10.1006/qres. 1993.1067

Espizua, L.E., 1998, Secuencia glacial del Pleistoceno tardío en el Valle del Río Grande, Mendoza, Argentina: Fuentes Geográficas de Bamberg, 15, 85-99.

Espizua, L.E., 2005, Holocene glacier chronology of Valenzuela Valley, Mendoza Andes, Argentina: The Holocene 17 (7), 1079-1085. https://doi. org/10.1191/0959683605hl866rr

Espizua, L.E., Bengochea, J.D., 2002, Landslide Hazard and Risk Zonation Mapping in the Río Grande Basin, Central Andes of Mendoza, Argentina: Mountain Research and Development, 22 (2), 177-185. https:// doi.org/10.1659/0276-4741(2002)022[0177:LH ARZM]2.0.CO;2

Espizua, L.E., Bigazzi, J., Iunes, P.J., Hadler, J.C., Osorio, A.M., 2002, Fission-track dating of a tephra layer related to Poti-Malal and Seguro drifts in the Río Grande basin, Mendoza, Argentina: Journal of Quaternary Science, 17 (8), 781-788. https://doi.org/10.1002/ jqs. 723

Espizua, L., Pitte, P., 2009, The Little Ice Age glaciar advance in the Central Andes $\left(35^{\circ}\right.$ S), Argentina: Palaeogeography,
Palaeoclimatology, Palaeoecology, 281 (34), 345-350. https://doi.org/10.1016/j. palaeo.2008.10.032

Farías, M., Tapia, F., Comte, D., 2009, La Falla Calabozos: Un cabalgamiento activo en la alta cordillera de Curicó (resumen electrónico), en XII Congreso Geológico Chileno, Santiago, Chile.

Fauqué, L, Hermanns R., Hewitt K., Rosas M., Wilson C., Baumann V., Lagorio S., Di Tommasso I., 2009, Megadeslizamientos de la pared sur del cerro Aconcagua y su relación con la génesis del depósito de Horcones. Mendoza, Argentina: Revista de la Asociación Geológica Argentina, 65 (4), 691-712.

Fauqué, L., Hermanns, R.L., Wilson, C., Rosas, M., Tedesco, A.M., Miranda, F., 2014, Revisión de la estratigrafía glaciaria de la cuenca del río Mendoza (resumen), en $19^{\circ}$ Congreso Geológico Argentino, Córdoba, S13-2.

Folguera, A., Bottessi, G., Zapata, T., Ramos, V.A., 2008, Crustal collapse in the Andean backarc since 2 Ma: Tromen volcanic plateau, Southern Central Andes (36 $40^{\prime}$ - 37 $30^{\prime}$ S): Tectonophysics, 459, 140-160. https://doi. org/10.1016/j.tecto.2007.12.013

Folguera, A., Naranjo, J., Orihashi, Y., Sumino, H., Nagao, K., Polanco, E., Ramos, V.A., 2009, Retroarc volcanism in the northern San Rafael Block (34은 $35^{\circ} 30^{\prime}$ 'S), southern Central Andes: Occurrence, age, and tectonic setting: Journal of Volcanology and Geothermal Research, 186, 169-185. https://doi. org/10.1016/j.jvolgeores.2009.06.012

Folguera, A., Bottesi, G., Duddy, I., MartínGonzález, F., Orts, D., Sagripanti, L., Rojas Vera, E., Ramos, V.A., 2015, Exhumation of the Neuquén Basin in the southern Central Andes (Malargüe fold and thrust belt) from field data and lowtemperature thermochronology: Journal of South American Earth Sciences, 64, 381-398. https://doi.org/10.1016/j. jsames.2015.08.003 
Gansser, A., 1973, Facts and theories on the Andes: Journal of the Geological Society, 129, 93-131. https://doi.org/10.1144/ gsjgs. 129.2.0093

Giambiagi, L., Bechis, F., Álvarez, P.P., Tunik, M.A., 2005, Influencia de las estructuras de rift triásico-jurásicas sobre el estilo de deformación en las fajas plegadas y corridas de Aconcagua y Malargüe, Mendoza: Revista de la Asociación Geológica Argentina, 60 (4), 662-671.

Giambiagi, L. B., Bechis, F., García, V., Clark, A., 2008, Temporal and spatial relationships of thick- and thin-skinned deformation: A case study from the Malargüe fold-and-thrust belt, southern Central Andes: Tectonophysics, 459, 123-139. https://doi.org/10.1016/j. tecto.2007.11.069

Giambiagi, L., Ghiglione, M., Cristallini, E., Bottesi, G., 2009, Características estructurales del sector sur de la faja plegada y corrida de Malargüe (35-36 $\mathrm{S})$ : Distribución del acortamiento e influencia de estructuras previas: Revista de la Asociación Geológica Argentina 65, 140-153.

Giambiagi, L., Álvarez, P., Spagnotto, S., Godoy, E., Lossada, E., Mescua, J., Barrionuevo, M., Suriano, J., 2019, Geomechanical model for a seismically active geothermal field: Insights from the Tinguiririca volcanic-hydrothermal system: Geoscience Frontiers, 10, 2117-2133. https://doi.org/10.1016/j.gsf.2019.02.006

Groeber, P., 1946, Observaciones Geológicas a lo largo del Meridiano 70 . 1 Hoja Chos Malal, Buenos Aires: Revista de la Asociación Geológica Argentina, 1 (3), 177-208

Grube, A, 2019, Palaeoseismic structures in Quaternary sediments, related to an assumed fault zone north of the Permian Peissen-Gnutz salt structure (NW Germany) - Neotectonic activity and earthquakes from the Saalian to the Holocene: Geomorphology, 328, 1, 15-27. https://doi.org/10.1016/j. geomorph.2018.12.004

Hungr, O., Leroueil, S., Picarelli, L., 2014, The Varnes classification of landslide types, an update: Landslides, 11 (2), 167-194. https:// doi.org/10.1007/s10346-013-0436-y

Keefer, D.K., 1984, Landslides caused by earthquakes: Bulletin of the Geological Society of America, 95, 406-421. https:// doi.org/10.1130/0016-7606(1984)95\&amp; lt;406:LCBE\&amp;gt;2.0.CO;2

Kozlowski, E., Manceda, R., Ramos, V.A., 1993, Estructura, en Ramos V. A. (ed.), Geología y recursos naturales de Mendoza, Relatorio. $12^{\circ}$ Congreso Geológico Argentino y $2^{\circ}$ Congreso Geológico de Exploración de Hidrocarburos, Mendoza, 1 (18), 235-256.

Legarreta, L., Uliana, M.A., 1999, El Jurásico y Cretácico de la Cordillera Principal y la cuenca Neuquina. 1. Facies sedimentarias, en Caminos, R. (ed.), Geología Argentina, Buenos Aires, Servicio Geológico y Minero Argentino, Instituto de Geología y Recursos Minerales, Anales 29 (16), 399-416.

Lúnkenheimer, F., 1929, El terremoto surmendocino del 30 de mayo de 1929. Contribuciones Geofísicas, Observatorio Astronómico de la Universidad Nacional de La Plata, Tomo III.

Maltman A., 1994, Deformation structures preserved in rocks, en Maltman A. (eds), The Geological Deformation of Sediments. Dordrecht. Springer, 261-302. https://doi. org/10.1007/978-94-011-0731-0_9

Mescua, J., Giambiagi, L., Ramos, V., 2013, Late Cretaceous Uplift in the Malargüe fold-andthrust belt $\left(35^{\circ} \mathrm{S}\right)$, southern Central Andes of Argentina and Chile: Andean Geology, 40 (1), 102-116. http://dx.doi.org/10.5027/ andgeoV40n 1-a05

Mescua, J.F., Barrionuevo, M., Giambiagi, L., Suriano, J., Spagnotto, S., Stahlschmidt, E., De la Cal, H., Soto, J.L., Mazzitelli, M., 2019, Stress field and active faults in the orogenic front of the Andes in the Malargüe foldand-thrust belt $\left(35^{\circ}-36^{\circ} \mathrm{S}\right)$ : Tectonophysics, 766, 179-193. https://doi.org/10.1016/j. tecto.2019.06.003

Moretti, M., Pieri, P., Tropeano, M., Walsh, N., 1995, Tyrrhenian seismites in Bari area 
(Murge-Apulian foreland) (resumen), en Atti dei Convegni Terremoti in Italia, Roma, Accademia Nazionale dei Lincei, 211-216.

Obermeier, S., 1994, Using liquefaction-induced features for paleoseismic analysis, en Obermeier, S., Gibson, W. (eds.), Using Ground-failure Features for Paleoseismic Analysis, Geological Survey Open-File Report, 94-633, A1-A98. https://doi. org/10.1016/S0013-7952(96)00040-3

Owen, G., 2003, Load structures: gravitydriven sediment mobilization in the shallow subsurface, en Van Rensbergen, P., Hillis, R.R., Maltman, A.J., Moreley, G.K. (eds.), Subsurface Sediment Mobilization. London, Geological Society, Special Publications, 216, 21-34. https://doi.org/10.1144/GSL. SP.2003.216.01.03

Pisarska-Jamroży, M., Belzyt, S., Börner, A., Hoffmann, G., Hüneke, H., Kenzler, M., Obst, K., Rother, H., van Loon, A., J., 2018, Evidence from seismites for glacioisostatically induced crustal faulting in front of an advancing land-ice mass (Rügen Island, SW Baltic Sea): Tectonophysics, 745, 338-348. https://doi.org/10.1016/j. tecto.2018.08.004

Ramos, V.A., 1999, Rasgos estructurales del territorio argentino, en Caminos, R. (ed.), Geología Argentina, Buenos Aires, Instituto de Geología y Recursos Minerales, Anales 29, 715-784.

Ramos, V.A., Kay, S.M., 2006, Overview of the tectonic evolution of the southern Central Andes of Mendoza and Neuquén $\left(35^{\circ}-39^{\circ} \mathrm{S}\right.$ latitude), en Kay, S.M., Ramos, V.A. (eds.), Evolution of an Andean Margin: A Tectonic and Magmatic View from the Andes to the

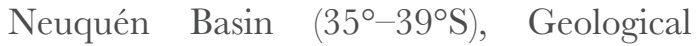
Society of America Special Paper, 407, 1-18. https://doi.org/10.1130/2006.2407(01)

Rodríguez, C.E., Bommer, J.J., Chandler, R.J., 1999, Earthquake-induced landslides: 1980-1997: Soil Dynamics and Earthquake Engineering, 18 (5), 325-346. https://doi. org/10.1016/S0267-7261(99)00012-3
Santibáñez, I., Cembrano, J., García-Pérez, T. Costa, G., Yáñez, G., Marquardt, G., Arancibia, G., González, G., 2019, Crustal faults in the Chilean Andes: geological constraints and seismic potential: Andean Geology, 46, 32-65. https://doi. org/10.5027/andgeoV46n1-3067

Seed, H., 1969, Landslides during earthquakes due to solid liquefaction: Journal of the Soil Mechanics Foundations Division, 95(4), 1053-1122. https://doi.org/10.1061/ jsfeaq.0001313

Silvestro, J., Kraemer, P., Achilli, F., Brinkworth, W., 2005, Evolución de las cuencas sinorogénicas de la Cordillera Principal entre $35^{\circ}-36^{\circ} \mathrm{S}$, Malargüe: Revista de la Asociación Geológica Argentina, 60 (4), 627-643.

Sims, J.D., 2013, Earthquake-induced load casts, pseudonodules, ball-and-pillow structures, and convolute lamination: Additional deformation structures for paleoseismic studies, en Cox, R.T., Tuttle, M.P., Boyd, O.S., Locat, J., (eds.), Recent Advances in North American Paleoseismology and Neotectonics East of the Rockies, Geological Society of America Special Paper, 493, 191-201. https://doi.org/10.1 130/2012.2493(09)

Spagnotto, S., Triep, E., Giambiagi, L., Lupari, M., 2015, Triggered seismicity in the Andean arc region via static stress variation by the $\mathrm{Mw}$ 8.8, February 27, 2010, Maule Earthquake: Journal of South American Earth Sciences, 63, 36- 47. https://doi.org/10.1016/j. jsames.2015.06.009

Sruoga, P. Gozalvez, M. Marquetti, C. Etcheverría, M.P. Mescua, J.F. Jara, A. Iannizzotto, N. Singer, B.S. Jicha, B.R., 2020, Early stages of the Miocene magmatic arc and related hydrothermal alteration at Valle Hermoso, South Central Andes ( $35^{\circ} 07^{\prime}$ S, $\left.70^{\circ} 17^{\prime} \mathrm{W}\right)$ : Journal of South American Earth Sciences, 99,102508. https://doi.org/10.1016/j. jsames.2020.102508

Tapia, F., Farías, M., Naipauer, M., Puratich, J., 2015, Late Cenozoic contractional evolution 
of the current arc-volcanic region along the southern Central Andes (35²0’S): Journal of Geodynamics, 88, 36-51. https://doi. org/10.1016/j.jog.2015.01.001

UNCuyo, 2017, Estudio Integrado de Impacto Ambiental(EsIIA) dela obra aprovechamiento Multipropósito Portezuelo del Viento (AMPDV). Reporte Técnico, Tomo II, 432 p.

Van Loon, A.J., Pisarska-Jamrozy, M., Nartiss, M., Krievans, M., Soms, J., 2016, Seismites resulting from high-frequency, highmagnitude earthquakes in Latvia caused by Late Glacial glacio-isostatic uplift: Journal of Palaeogeography, 5(4), 363-380. https://doi. org/10.1016/j.jop.2016.05.002

Varnes, D.J., 1978, Slope movement types and processes, en Schuster R.L., Krizek, R.J. (eds), Landslides: Analysis and control, Transportation Research Board, Washington, DC, National Academy of Sciences, Special
Report, 176, 11-33.

Villegas, A., Raquel, J., Zahradnik, J., Nacif, S., Spagnotto, S., Winocur, D., Leiva, M., 2016, Waveform inversion and focal mechanisms of two weak earthquakes in Cordillera Principal (Argentina) between $35^{\circ}$ and $35.5^{\circ} \mathrm{S}$ : Journal of South American Earth Sciences, 71, 359-369. https://doi.org/10.1016/j. jsames.2015.12.001

Yrigoyen, M., 1979, Cordillera Principal, en $2^{\circ}$ Simposio de Geología Regional Argentina, Córdoba, Academia Nacional de Ciencias, 953-984.

Zech, J., Terrizzano, G., García-Morabito, E., Veit, H., Zech, R., 2017, Timing and extent of Late Pleistocene glaciation in the arid Central Andes of Argentina and Chile $\left(22^{\circ}-41^{\circ} \mathrm{S}\right)$ : Cuadernos de Investigación Geográfica, 43(2), 697-718. https://doi. org/10.18172/cig.3235 